\title{
On a New Modification of the Weibull Model with Classical and Bayesian Analysis
}

\author{
Yen Liang Tung, ${ }^{1}$ Zubair Ahmad ${ }^{D},{ }^{2}$ Omid Kharazmi, ${ }^{3}$ Clement Boateng Ampadu, ${ }^{4}$ \\ E.H. Hafez, ${ }^{5}$ and Sh. A.M. Mubarak ${ }^{6}$ \\ ${ }^{1}$ Accounting Department, School of Business, Nanjing University, Nanjing 210093, China \\ ${ }^{2}$ Department of Statistics, Yazd University, P.O. Box 89175-741,, Yazd, Iran \\ ${ }^{3}$ Department of Statistics, Faculty of Sciences, Vali-e-Asr University of Rafsanjan, Rafsanjan, Iran \\ ${ }^{4}$ Department of Mathematics, Central Michigan University, Mt Pleasant 48859, MI, USA \\ ${ }^{5}$ Department of Mathematics, Faculty of Science, Helwan University, Cairo, Egypt \\ ${ }^{6}$ High Institute of Engineering and Technology, Ministry of Higher Education, El-Minia, Egypt \\ Correspondence should be addressed to Zubair Ahmad; z.ferry21@gmail.com
}

Received 18 January 2021; Revised 7 February 2021; Accepted 19 February 2021; Published 4 March 2021

Academic Editor: Ahmed Mostafa Khalil

Copyright (c) 2021 Yen Liang Tung et al. This is an open access article distributed under the Creative Commons Attribution License, which permits unrestricted use, distribution, and reproduction in any medium, provided the original work is properly cited.

\begin{abstract}
Modelling data in applied areas particularly in reliability engineering is a prominent research topic. Statistical models play a vital role in modelling reliability data and are useful for further decision-making policies. In this paper, we study a new class of distributions with one additional shape parameter, called a new generalized exponential- $X$ family. Some of its properties are taken into account. The maximum likelihood approach is adopted to obtain the estimates of the model parameters. For assessing the performance of these estimators, a comprehensive Monte Carlo simulation study is carried out. The usefulness of the proposed family is demonstrated by means of a real-life application representing the failure times of electronic components. The fitted results show that the new generalized exponential- $X$ family provides a close fit to data. Finally, considering the failure times data, the Bayesian analysis and performance of Gibbs sampling are discussed. The diagnostics measures such as the Raftery-Lewis, Geweke, and Gelman-Rubin are applied to check the convergence of the algorithm.
\end{abstract}

\section{Introduction}

Generally speaking, lifetime distributions have been frequently applied to model lifetime data in many fields especially in reliability engineering and biomedical sciences. Due to the variability of the data, the selection of the statistical models greatly affects the quality of the modelling to provide the best description of the phenomena under consideration, for instance, data modelling with the exponential and Rayleigh models when the data experience the decreasing failure rate, or the utilization of the Rayleigh model when the data has a constant failure behaviour. Henceforth, it is always of interest to provide the best fit to data under consideration. In such situations, the utilization of the Weibull model may be a suitable choice to analyze data having increasing, decreasing, or constant failure rates, for example, modified Weibull distribution [1], beta modified Weibull distribution [2], and a new modified Weibull distribution [3].

However, in a number of situations, where data behaves nonmonotonically such as unimodal, modified unimodal, or bathtub shaped failure rates, then the Weibull model is not a good candidate model to use. So, for accurate and precise data modelling, new extensions and modifications of the existing models are required. Therefore, many statistical methods are designed to find new extensions of the existing models to provide a better fit to the data of interest.

In the literature, most of the modifications of the Weibull model have been derived by introducing new families of distributions; see the beta extended Weibull family [4], the 
Weibull-G family [5], a new Weibull- $X$ family [6]. For a more brief review, we refer to Ahamd et al. [7].

We are also continuing this research area and proposing a new statistical distribution family, namely, a new generalized exponential- $X$ (NGE- $X$ ) family of distributions.

Genesis: let $p(t)$ be the probability density function (pdf) of a random variable, say $T$, where $T \in\left[\kappa_{1}, \kappa_{2}\right]$, $-\infty \leq \kappa_{1}<\kappa_{2}<\infty$, and let $W[F(x)]$ be a function of cumulative distribution function (cdf) $F(x)$ of a random variable, say $X$, satisfying the conditions given below:

(1) $W[F(x)] \in\left[\kappa_{1}, \kappa_{2}\right]$

(2) $W[F(x)]$ is differentiable and monotonically increasing

(3) $W[F(x)] \longrightarrow \kappa_{1}$ as $x \longrightarrow-\infty$ and $W[F(x)]$ $\longrightarrow \kappa_{2}$ as $x \longrightarrow \infty$ by

The cdf of the T-X family of distributions [8] is defined

$$
G(x)=\int_{\kappa_{1}}^{W[F(x)]} p(t) \mathrm{d} t, \quad x \in \mathbb{R},
$$

where $W[F(x)]$ satisfies the conditions stated above. The pdf corresponding to (1) is

$$
g(x)=\left\{\frac{\partial}{\partial x} W[F(x)]\right\} v\{W[F(x)]\}, \quad x \in \mathbb{R} .
$$

Using the approach of the T- $X$ method, one can introduce new members of the survival family via the cdf given by

$$
G(x)=1-\int_{\kappa_{1}}^{W[\bar{F}(x)]} v(t) \mathrm{d} t, \quad x \in \mathbb{R},
$$

where $\bar{F}(x)=1-F(x)$ is the survival function of the baseline distribution.

Taking inspiration from (1), we introduce a new flexible class of distributions, namely, a new generalized exponential- $X$ (NGE- $X)$ family of distributions. Let $T \sim \exp (1)$; then, its cdf is given by

$$
P(t)=1-e^{-t}, \quad t \geq 0 .
$$

The density function corresponding to (4) is

$$
p(t)=e^{-t}, \quad t>0 \text {. }
$$

If $p(t)$ follows (5) and setting $W[F(x)]=-\log ([1-$ $\left.\left.F(x ; \xi)^{2}\right]^{\theta} / e^{\theta F(x ; \xi)^{2}}\right)$ in (1), we define the cdf of the NGE- $X$ family given by

$$
G(x ; \theta, \xi)=1-\left(\frac{\left[1-F(x ; \xi)^{2}\right]^{\theta}}{e^{\theta F(x ; \xi)^{2}}}\right), \quad x \in \mathbb{R} .
$$

The corresponding pdf is

$$
g(x ; \theta, \xi)=\frac{2 \theta f(x ; \xi) F(x ; \xi)\left[1-F(x ; \xi)^{2}\right]^{\theta-1}}{e^{\theta F(x ; \xi)^{2}}}\left\{2-F(x ; \xi)^{2}\right\}, \quad x \in \mathbb{R} .
$$

Based on the proposed procedure defined in (6), a special case is being studied, namely, a new generalized exponentialWeibull (NGE-Weibull), in the belief that it will be most effective in all areas where the Weibull model is applicable. The new distribution is a flexible model that is able to play an important role in reliability analysis as it can take on a variety of shapes of the failure rate function.

Furthermore, we consider the maximum likelihood and Bayesian approaches in order to estimate the parameters of the model. In the Bayesian discussion, we consider different types of symmetric and asymmetric loss functions including weighted squared error, squared error loss, precautionary, $K$-loss, and modified squared error loss function to estimate the unknown parameters of the NGE-Weibull model. Since all the parameters are positive, we use gamma prior distributions. Bayesian 95\% credible and highest posterior density (HPD) intervals [9] are given for each of the proposed model parameters. The posterior samples were extracted via the Gibbs sampling process. From the graphical point of view, we sketch the posterior summary plots. Next, to explore the MCMC process in Bayesian analysis, we used the Gelman-Rubin, Geweke, and Raftery-Lewis diagnostic methods for testing the convergence of the algorithm.

The remaining sections of the article are organized as follows: Section 2 offers the proposed model with its graphical illustrations. In Section 3, the statistical properties are obtained. Section 4 is devoted to the parameter estimation by maximum likelihood estimation (MLE) and Monte Carlo simulation study. In Section 5, the proposed distribution is illustrated by analyzing the failure time data. The Bayesian analysis is provided in Section 6. Finally, this research is concluded in the last section.

\section{Submodel Description}

The two-parameter Weibull distribution $(\xi=\alpha, \gamma)$ has pdf, cdf, survival function (sf), hazard rate function (hrf), and cumulative hazard rate function (chrf) given by $f(x ; \xi)=\alpha \gamma x^{\alpha-1} e^{-\gamma x^{\alpha}}, F(x ; \xi)=1-e^{-\gamma x^{\alpha}}, S(x ; \xi)=e^{-\gamma x^{\alpha}}$, $h(x ; \xi)=\alpha \gamma x^{\alpha-1}$ and $H(x ; \xi)=\gamma x^{\alpha}$, respectively. Then, the cdf and pdf of the NGE-Weibull distribution are given by (for $x>0$ ) 


$$
\begin{aligned}
& G(x ; \theta, \xi)=1-\left(\frac{\left[1-\left(1-e^{-\gamma x^{\alpha}}\right)^{2}\right]^{\theta}}{e^{\theta\left(1-e^{-\gamma x^{\alpha}}\right)^{2}}}\right), \\
& g(x ; \theta, \xi)=\frac{2 \theta \alpha \gamma x^{\alpha-1} e^{-\gamma x^{\alpha}}\left(1-e^{-\gamma x^{\alpha}}\right)\left[1-\left(1-e^{-\gamma x^{\alpha}}\right)^{2}\right]^{\theta-1}}{\left.e^{\theta\left(1-e^{-\gamma x^{\alpha}}\right.}\right)^{2}}\left\{2-\left(1-e^{-\gamma x^{\alpha}}\right)^{2}\right\},
\end{aligned}
$$

respectively.

The NGE-Weibull density and hazard rate plots for chosen parameter values are provided, respectively, in Figures 1 and 2.

\section{Mathematical Properties}

This section is devoted to deriving the mathematical properties of the NGE- $X$ distributions including the quantile function, expansions of $\mathrm{cdf}$ and pdf, reliability, random number generation, $r^{\text {th }}$ noncentral moments, and entropy with numerical illustrations. Furthermore, a characterization theorem extending the NGE- $X$ class of distributions is also derived.

3.1. Quantile Function. Letting $0<p<1$, we must solve the following equation for $Q(p)$ :

$$
p=1-\frac{\left(1-F(Q(p))^{2}\right)^{\theta}}{e^{\theta F(Q(p))^{2}}} .
$$

Letting $y=F(Q(p))$, then solving the following equation for $y$ (using software like MATHEMATICA)

$$
p=1-\frac{\left(1-y^{2}\right)^{\theta}}{e^{\theta y^{2}}},
$$

it can be shown that

$$
F(Q(p))=\sqrt{1-W\left(\left(-e^{\theta}(p-1)\right)^{1 / \theta}\right)},
$$

where $W(z)$ gives the principal solution for $m$ in $z=m e^{m}$. Thus,

$$
Q(p)=F^{-1}\left\{\sqrt{1-W\left(\left(-e^{\theta}(p-1)\right)^{1 / \theta}\right)}\right\},
$$

where $F^{-1}$ is the quantile of the baseline distribution with $c d f$ $F(x)$.

Remark 1. Throughout, we assume the Weibull distribution has quantile:

$$
Q(x, a, b)=b(-\log (1-x))^{1 / a},
$$

where $a, b>0$ and $0<u<1$. Whenever the baseline distribution is Weibull, we refer to the submodel as $\operatorname{NGE}$ - Weibull $(\theta, a, b)$.

Some numerical values of the quantile measure are provided in Table 1.
The quantile function is used to measure the effect of the shape parameters on the skewness and kurtosis. Henceforth, using the quantile function of the NGE-Weibull distribution, we obtained the expressions for skewness and kurtosis. The formulas for Bowley's skewness and Moor's kurtosis are given

$$
\begin{aligned}
\text { skewness } & =\frac{Q(1 / 4)+Q(3 / 4)-2 Q(1 / 2)}{Q(3 / 4)-Q(1 / 4)}, \\
\text { kurtosis } & =\frac{Q(7 / 8)-Q(5 / 8)+Q(3 / 8)-Q(1 / 8)}{Q(6 / 8)-Q(2 / 8)},
\end{aligned}
$$

receptively.

For $\gamma=1$ and different values of $\alpha$ and $\theta$, graphs for the skewness and kurtosis of the NGE-Weibull are sketched in Figures 3 and 4 .

3.2. Expansion for Distribution Function. Using the binomial theorem, we have

$$
\left(1-F(x)^{2}\right)^{\theta}=\sum_{k=0}^{\theta}\left(\begin{array}{l}
\theta \\
k
\end{array}\right)(-1)^{k} F(x)^{2 k} .
$$

Using the power series representation for the exponential function (EP), we have

$$
e^{-\theta F(x)^{2}}=\sum_{q=0}^{\infty} \frac{(-1)^{q} \theta^{q} F(x)^{2 q}}{q !} .
$$

Put

$$
\Omega_{k, q}=\left(\begin{array}{l}
\theta \\
k
\end{array}\right)(-1)^{k} \frac{(-1)^{q} \theta^{q}}{q !} .
$$

It now follows that the cdf can be expressed as

$$
1-\sum_{k=0}^{\theta} \sum_{q=0}^{\infty} \Omega_{k, q} F(x)^{2 k+2 q} .
$$

3.3. Expansion for Density Function. From the binomial theorem, we have

$$
\left(1-F(x)^{2}\right)^{\theta-1}=\sum_{k=0}^{\theta-1}\left(\begin{array}{c}
\theta-1 \\
k
\end{array}\right)(-1)^{k} F(x)^{2 k} .
$$

By the power series representation for the exponential function, we can write 

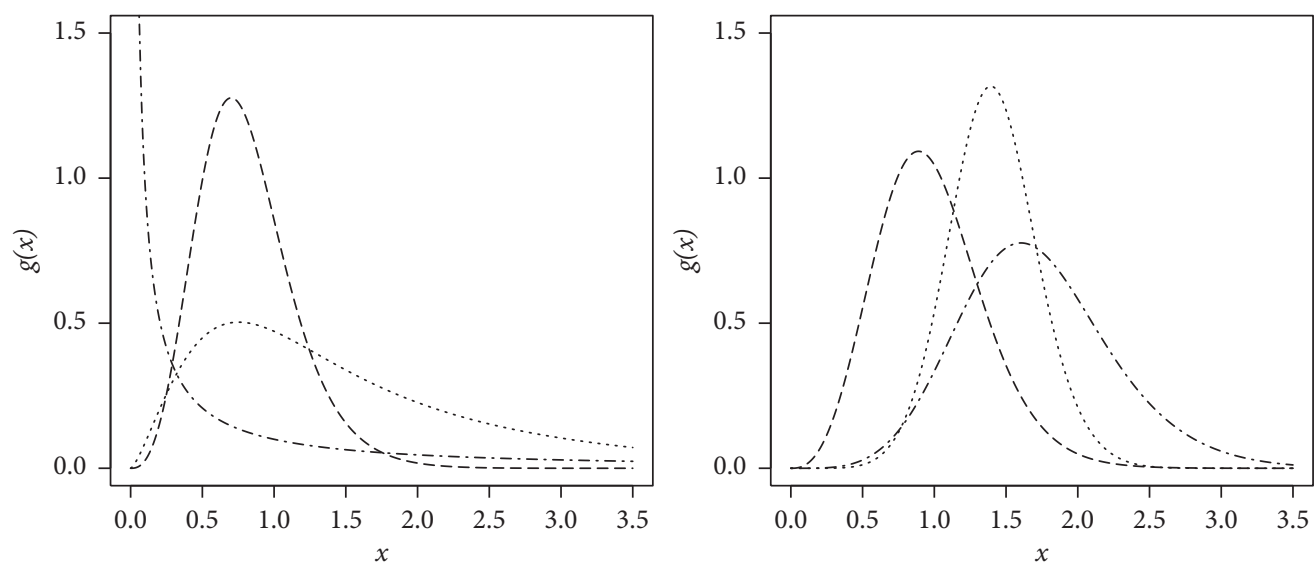

$$
\begin{aligned}
& \ldots \ldots \alpha=1.2, \theta=0.5, \gamma=1 \\
& ---\alpha=1.8, \theta=1.5, \gamma=1
\end{aligned}
$$$$
\text { … } \alpha=3.5, \theta=1.4, \gamma=0.2
$$$$
--\alpha=1.8, \theta=2.5, \gamma=0.5
$$$$
\text { -.. } \alpha=2.5, \theta=1.2, \gamma=0.2
$$

Figure 1: Density plots of the NGE-Weibull distribution.
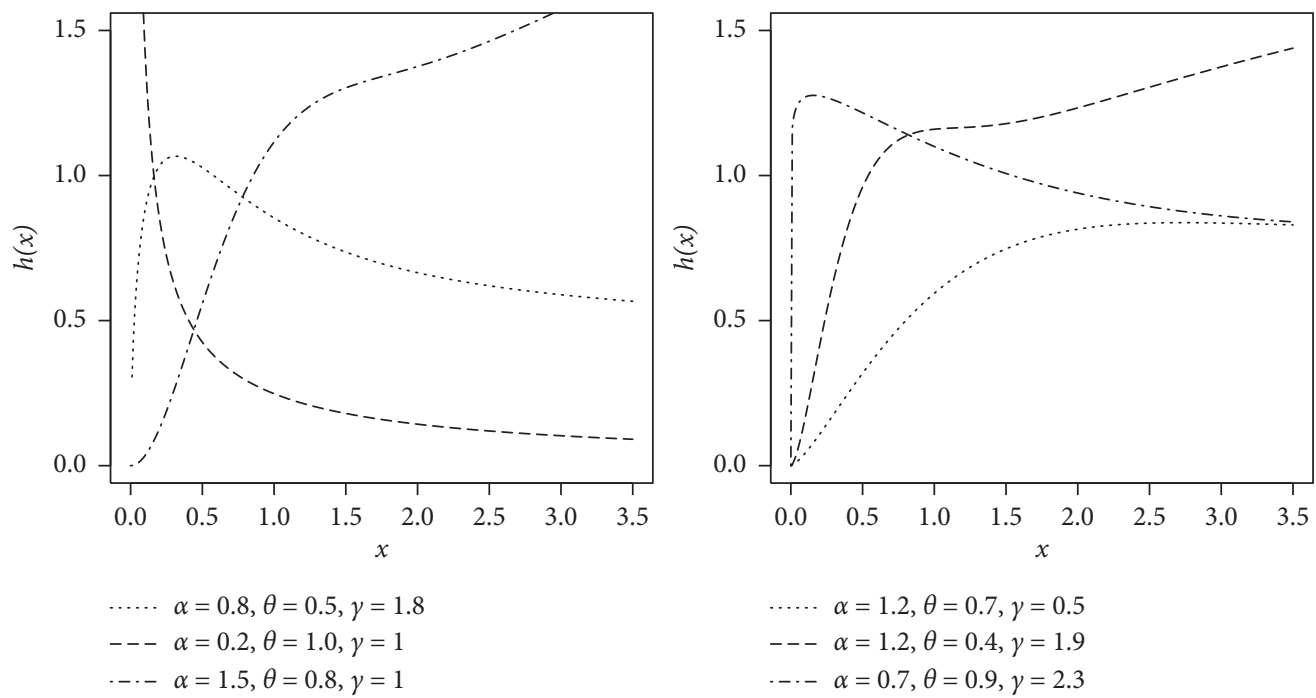

FIgURE 2: Hazard rate plots of the NGE-Weibull distribution.

TABle 1: Some quantile values.

\begin{tabular}{lr}
\hline$x$ & Q(x) of NGE-Weibull (3, 1, 1) \\
\hline 0.1 & 0.14182 \\
0.2 & 0.213124 \\
0.3 & 0.277044 \\
0.4 & 0.340545 \\
0.5 & 0.407744 \\
0.6 & 0.482993 \\
0.8 & 0.573055 \\
0.9 & 0.692116 \\
\hline
\end{tabular}




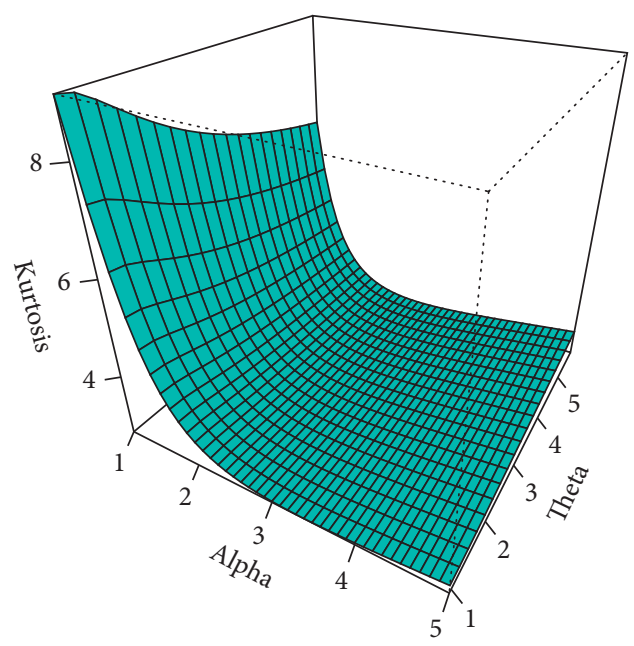

(a)

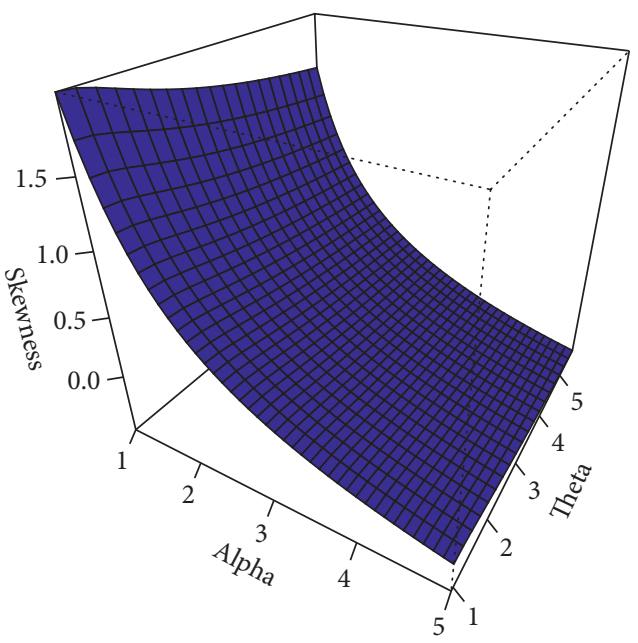

(b)

FIGURE 3: Plots for the skewness and kurtosis of the NGE-Weibull distribution.

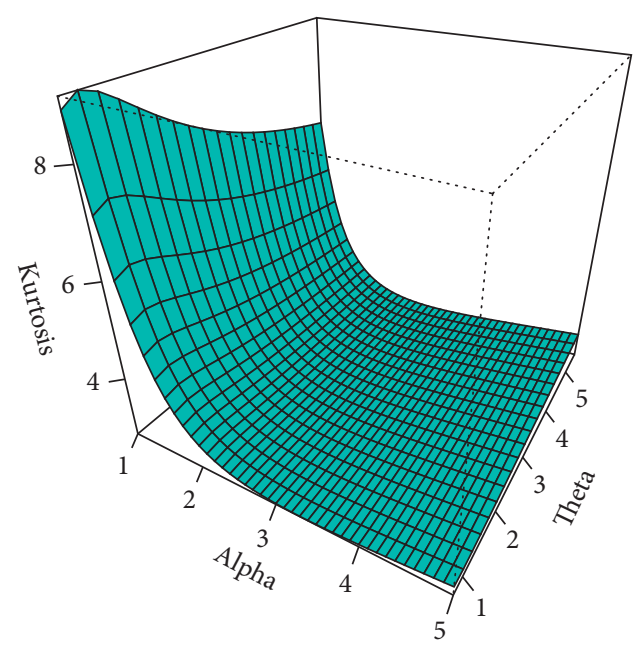

(a)

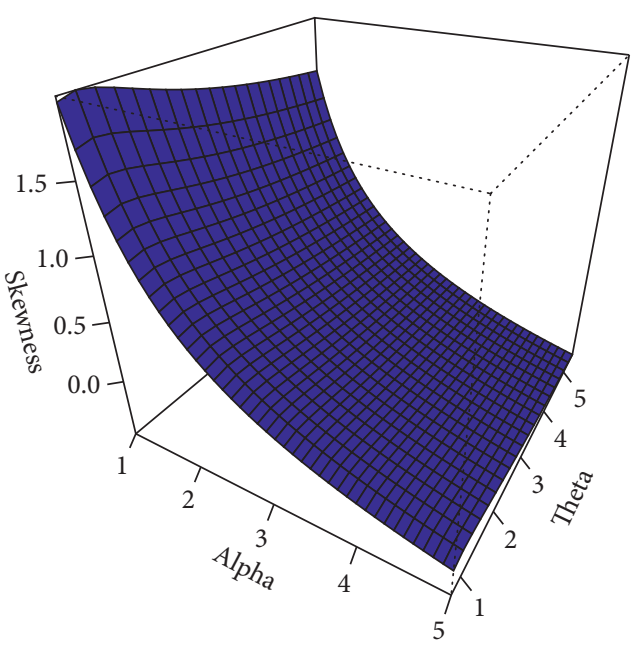

(b)

FIgURE 4: Plots for the skewness and kurtosis of the NGE-Weibull distribution.

$$
e^{-\theta F(x)^{2}}=\sum_{q=0}^{\infty} \frac{(-1)^{q} \theta^{q} F(x)^{2 q}}{q !} .
$$

Put

$$
\Omega_{k, q}=\left(\begin{array}{c}
\theta-1 \\
k
\end{array}\right) \frac{(-1)^{k+q} \theta^{q}}{q !} .
$$

It follows that we can write

$$
\left(1-F(x)^{2}\right)^{\theta-1} e^{-\theta F(x)^{2}}=\sum_{k=0}^{\theta-1} \sum_{q=0}^{\infty} \Omega_{k, q} F(x)^{2 k+2 q} .
$$

Finally, the expansion for the pdf is given as

$$
2 \theta f(x) \sum_{k=0}^{\theta-1} \sum_{q=0}^{\infty} \Omega_{k, q} F(x)^{2 k+2 q+1}\left(2-F(x)^{2}\right) .
$$

3.4. Reliability. In the concept of reliability theory, we know that the life of a component has a random strength with random stress. Random strength can be modelled by a random variable, say $Z$, and the random stress can be modelled by a random variable, say $Y$. The probability that the component works satisfactorily is $R=P(Y<Z)$, which is a known measure of component reliability for many applications. In particular,

$$
R=\int_{-\infty}^{\infty} k_{Z}(x) K_{Y}(x) \mathrm{d} x,
$$


where $k$ is a pdf and $K$ is a cdf.

Let $(\theta ; \xi)$ be a vector of parameters associated with the NGE- $X$ distributions, where $\xi$ is a vector of parameters associated with the distribution of the random variable $X$. If $Z$ is distributed as NGE- $X$ with parameter vector $\left(\theta_{1}, \xi_{1}\right)$ and $Y$ is distributed as NGE- $X$ with parameter vector $\left(\theta_{2}, \xi_{2}\right)$, then from the expansion of the pdf and cdf, we have

$$
R=\int_{-\infty}^{\infty} k_{Z}\left(x ; \theta_{1}, \xi_{1}\right) K_{Y}\left(x ; \theta_{2}, \xi_{2}\right) \mathrm{d} x,
$$

where $k_{Z}\left(x ; \theta_{1}, \xi_{1}\right)$ is given by

$$
2 \theta_{1} f\left(x ; \xi_{1}\right) \sum_{k=0}^{\theta_{1}-1} \sum_{q=0}^{\infty} \Omega_{k, q} F\left(x ; \xi_{1}\right)^{2 k+2 q+1}\left(2-F\left(x ; \xi_{1}\right)^{2}\right),
$$

with

$$
\Omega_{k, q}=\left(\begin{array}{c}
\theta_{1}-1 \\
k
\end{array}\right) \frac{(-1)^{k+q} \theta_{1}^{q}}{q !},
$$

and $K_{Y}\left(x ; \theta_{2}, \xi_{2}\right)$ is given by

$$
1-\sum_{m=0}^{\theta_{2}} \sum_{r=0}^{\infty} \Omega_{m, r} F\left(x ; \xi_{2}\right)^{2 m+2 r},
$$

with

$$
\Omega_{m, r}=\left(\begin{array}{c}
\theta_{2} \\
m
\end{array}\right)(-1)^{m} \frac{(-1)^{r} \theta_{2}^{r}}{r !} .
$$

3.5. Random Number Generation. Random numbers from the NGE- $X$ distributions can be obtained from

$$
X=F^{-1}\left\{\sqrt{1-W\left(\left(-e^{\theta}(U-1)\right)^{1 / \theta}\right)}\right\},
$$

where $F^{-1}$ is the quantile of the baseline distribution with $\mathrm{cdf}$ $F(x), W(z)$ gives the principal solution for $m$ in $z=m e^{m}$, $U \sim \operatorname{uniform}(0,1)$, and $\theta>0$.

3.6. The $r^{\text {th }}$ Noncentral Moments. We know the random variable:

$$
X=F^{-1}\left\{\sqrt{1-W\left(\left(-e^{\theta}(U-1)\right)^{1 / \theta}\right)}\right\},
$$

where $F^{-1}$ is the quantile of the baseline distribution with $\mathrm{cdf}$ $F(x), W(z)$ gives the principal solution for $m$ in $z=m e^{m}$, $U \sim$ uniform $(0,1)$, and $\theta>0$, following the NGE- $X$ family of distributions. According to Nasiru et al. [10], we can write

$$
Q_{X}(u)=\sum_{i=0}^{\infty} h_{i} u^{i}
$$

where the coefficients are suitably chosen real numbers that depend on the parameters of the $F(x)$ distribution. For a power series raised to a positive integer $r \geq 1$, we have

$$
\left(Q_{X}(u)\right)^{r}=\left(\sum_{i=0}^{\infty} h_{i} u_{i}\right)^{r}=\sum_{i=0}^{\infty} \delta_{r, i} u^{i},
$$

where $\delta_{r, i}$ are obtained from $\delta_{r, i}=\left(i h_{0}\right)^{-1} \sum_{s=1}^{i}[s(r+1)-i]$ $h_{s} \delta_{r, i-s}$ with $\delta_{r, 0}=h_{0}^{r}$ for $i=1,2, \ldots$; see Gradshteyn and Ryzhik [11]. Thus, we have the following:

$$
\left.\mu_{r}^{\prime}=\sum_{i=0}^{\infty} \delta_{r, i} E\left[\left(\sqrt{1-W\left(\left(-e^{\theta}(U-1)\right)^{1 / \theta}\right.}\right)\right)^{i}\right],
$$

where $E(\cdot)$ is an expectation. By the Binomial series, we can write

$$
\left(\sqrt{1-W\left(\left(-e^{\theta}(U-1)\right)^{1 / \theta}\right)}\right)^{i}
$$

as

$$
\sum_{k=0}^{\infty}\left(\begin{array}{c}
\frac{i}{2} \\
k
\end{array}\right)(-1)^{k} W\left[\left(-e^{\theta} U+e^{\theta}\right)^{(1 / \theta)}\right]^{k}
$$

By integer powers of the Lambert $\mathrm{W}$ function, we can write

$$
W\left[\left(-e^{\theta} U+e^{\theta}\right)^{(1 / \theta)}\right]^{k}
$$

as

$$
\sum_{n=k}^{\infty} \frac{-k(-n)^{n-k-1}}{(n-k) !}\left(-e^{\theta} U+e^{\theta}\right)^{(n / \theta)} .
$$

By the binomial series, we can write

$$
\left(-e^{\theta} U+e^{\theta}\right)^{(n / \theta)}=e^{n}(1-U)^{(n / \theta)},
$$

as

$$
e^{n} \sum_{m=0}^{\infty}\left(\begin{array}{c}
\bar{\theta} \\
m
\end{array}\right)(-1)^{m} U^{m}
$$

Put

$$
\Omega_{i, k, n, m}=\delta_{r, i}\left(\begin{array}{c}
\frac{i}{2} \\
k
\end{array}\right)(-1)^{k-k(-n)^{n-k-1}} \frac{(n-k) !}{(n-}\left(\begin{array}{c}
n \\
\bar{\theta} \\
m
\end{array}\right)(-1)^{m} .
$$

Thus, the $r^{\text {th }}$ noncentral moment is given by

$$
\mu_{r}^{\prime}=\sum_{i=0}^{\infty} \sum_{k=0}^{\infty} \sum_{n=k}^{\infty} \sum_{m=0}^{\infty} \Omega_{i, k, n, m} E\left[U^{m}\right] .
$$

Some numerical descriptions of the ordinary moments are presented in Table 2. 
TABLE 2: Some quantile values.

\begin{tabular}{lr}
\hline$x$ & $E\left[X^{r}\right]$ of NGE-Weibull $(3,1,1)$ \\
\hline 1 & 0.473155 \\
2 & 0.32289 \\
3 & 0.291456 \\
4 & 0.332991 \\
5 & 0.468629 \\
6 & 0.796351 \\
7 & 1.60647 \\
9 & 3.78649 \\
\hline
\end{tabular}

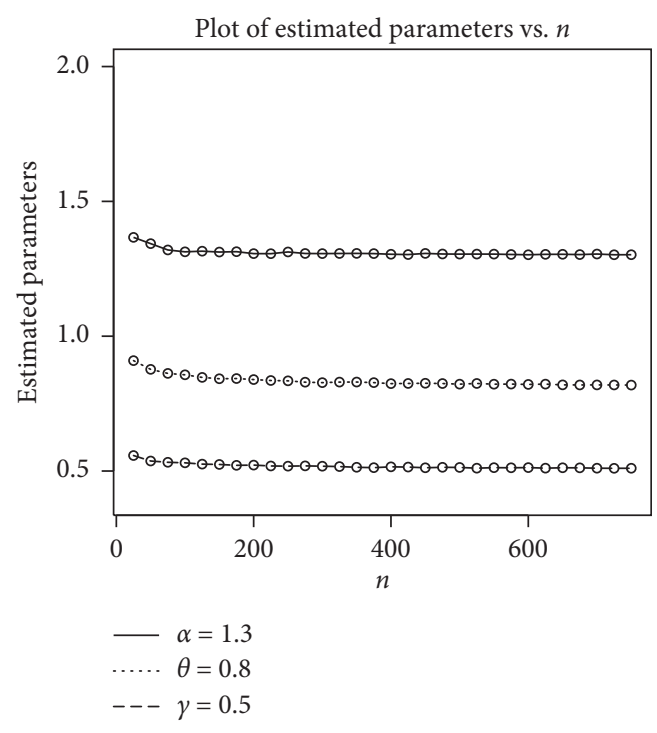

(a)

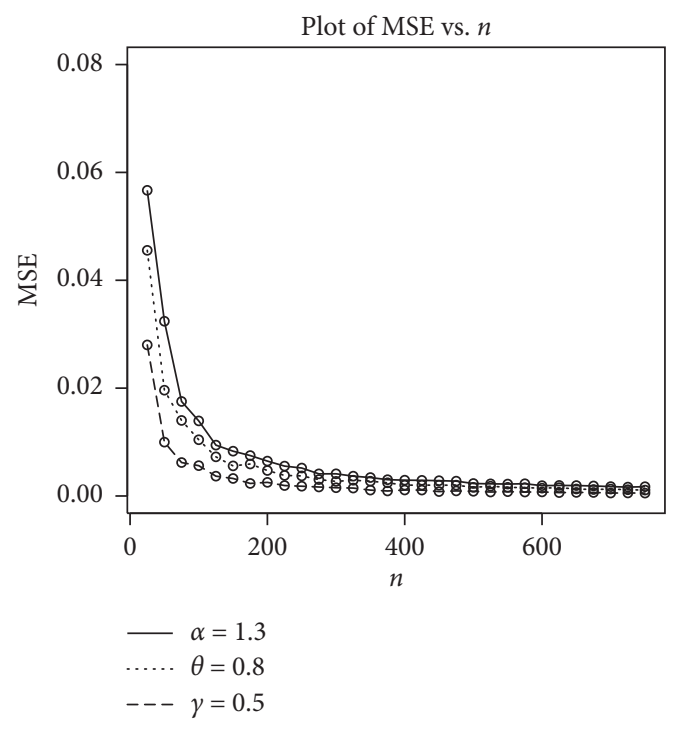

(b)

FIgUre 5: MLEs and MSEs plots of the NGE-Weibull distribution for $\alpha=1.3, \theta=0.8$, and $\gamma=0.5$.

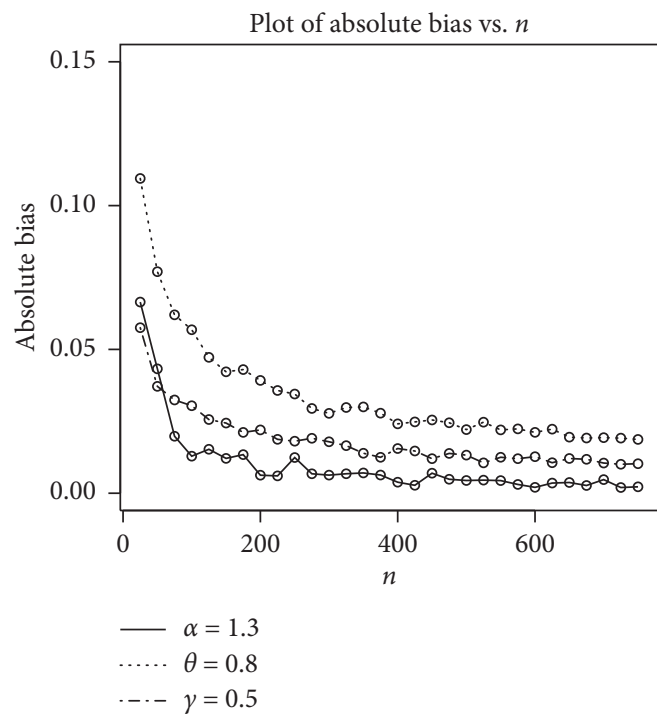

(a)

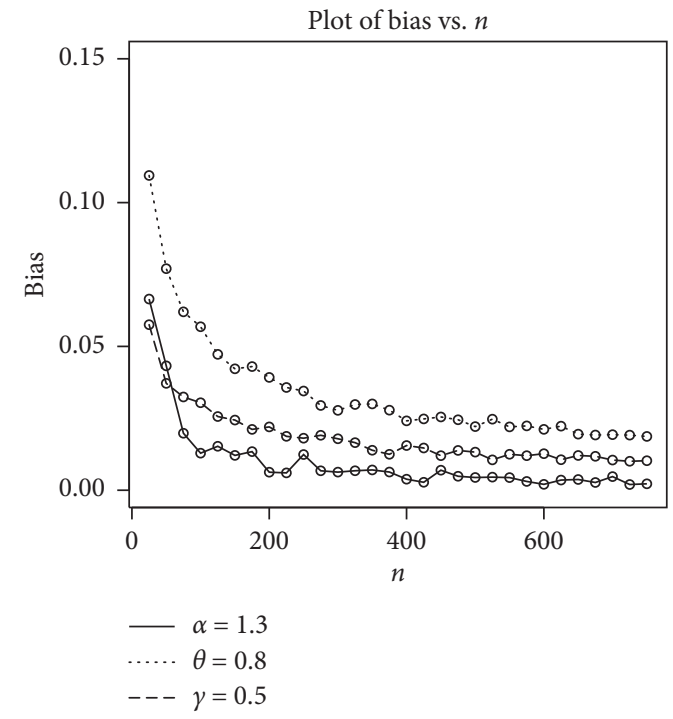

(b)

FIGURE 6: Biases and absolute biases plots of the NGE-Weibull distribution for $\alpha=1.3, \theta=0.8$, and $\gamma=0.5$. 


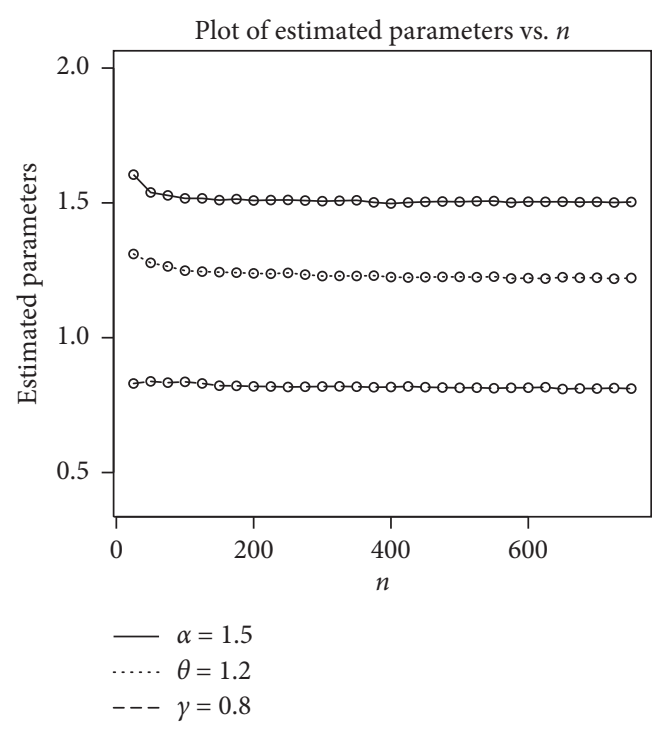

(a)

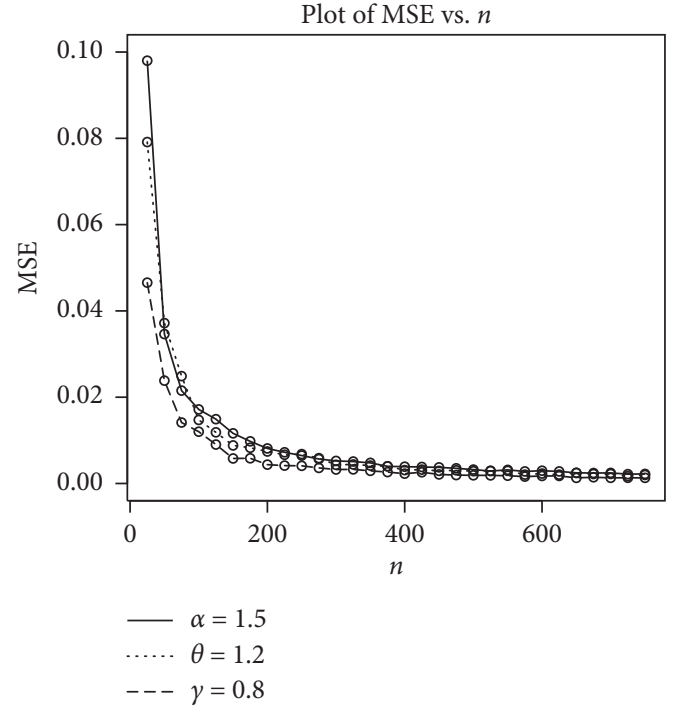

(b)

FIgURE 7: MLEs and MSEs plots of the NGE-Weibull distribution for $\alpha=1.5, \theta=1.2$, and $\gamma=0.8$.

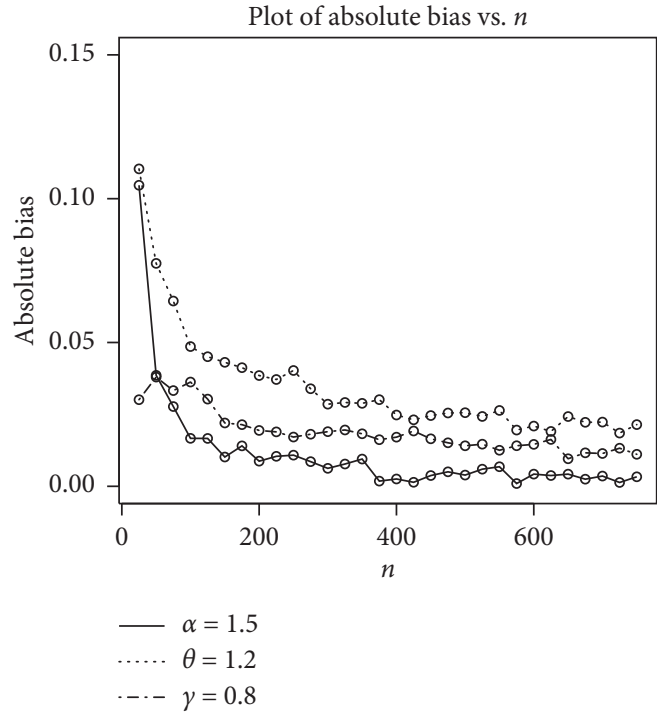

(a)

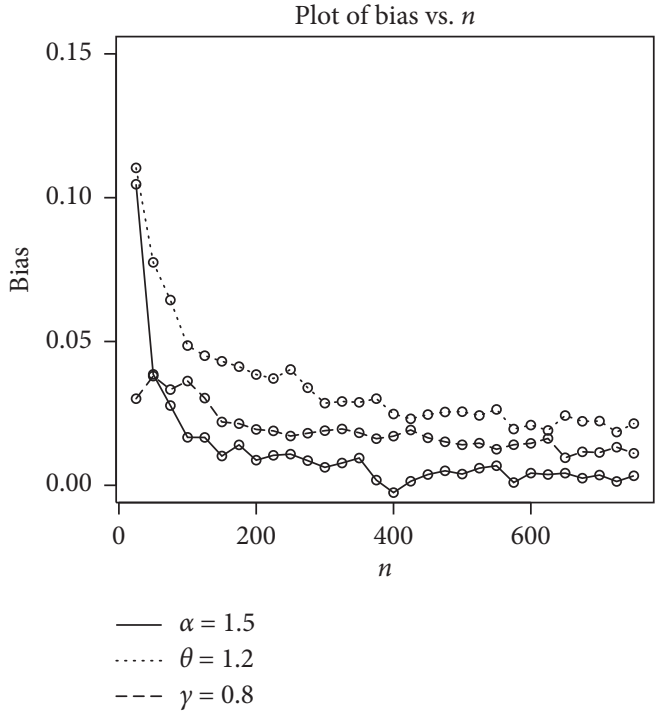

(b)

Figure 8: Biases and absolute biases plots of the NGE-Weibull distribution for $\alpha=1.5, \theta=1.2$, and $\gamma=0.8$.

3.7. Renyi Entropy. Using the binomial series, we can write $\left(1-F(x)^{2}\right)^{\delta(\theta-1)}$, as

$$
\sum_{k=0}^{\infty}\left(\begin{array}{c}
\delta(\theta-1) \\
k
\end{array}\right)(-1)^{k} F(x)^{2 k}
$$

Using the power series representation for the exponential function, we can write $e^{-\delta \theta F(x)^{2}}$, as

$$
\sum_{q=0}^{\infty} \frac{(-1)^{q} \delta^{q} \theta^{q} F(x)^{2 q}}{q !}
$$

By the binomial theorem, we can write $\left(2-F(x)^{2}\right)^{\delta}$ as

$$
\sum_{m=0}^{\delta}\left(\begin{array}{c}
\delta \\
m
\end{array}\right) 2^{\delta-m}(-1)^{m} F(x)^{2 m}
$$

Put

$\Omega_{k, q, m}=(2 \theta)^{\delta}\left(\begin{array}{c}\delta(\theta-1) \\ k\end{array}\right)(-1)^{k} \frac{(-1)^{q} \delta^{q} \theta^{q}}{q !}\left(\begin{array}{c}\delta \\ m\end{array}\right) 2^{\delta-m}(-1)^{m}$. 


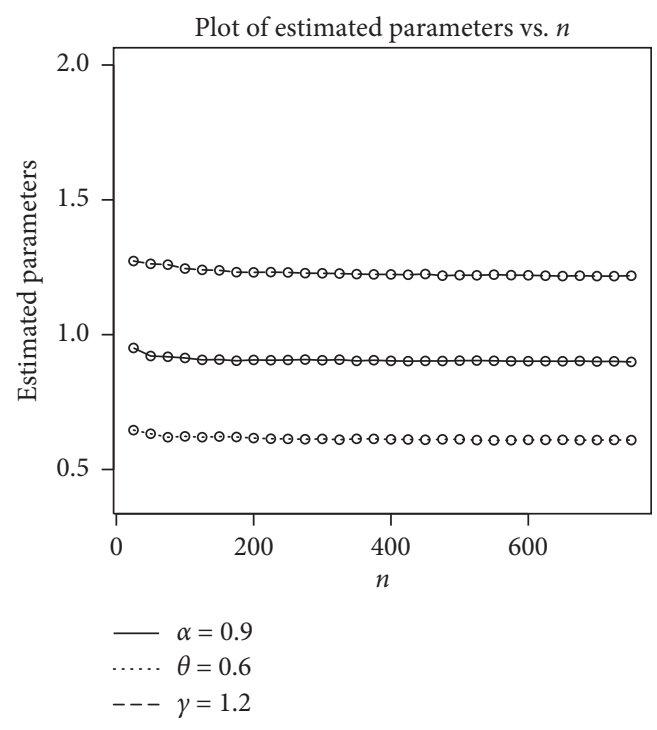

(a)

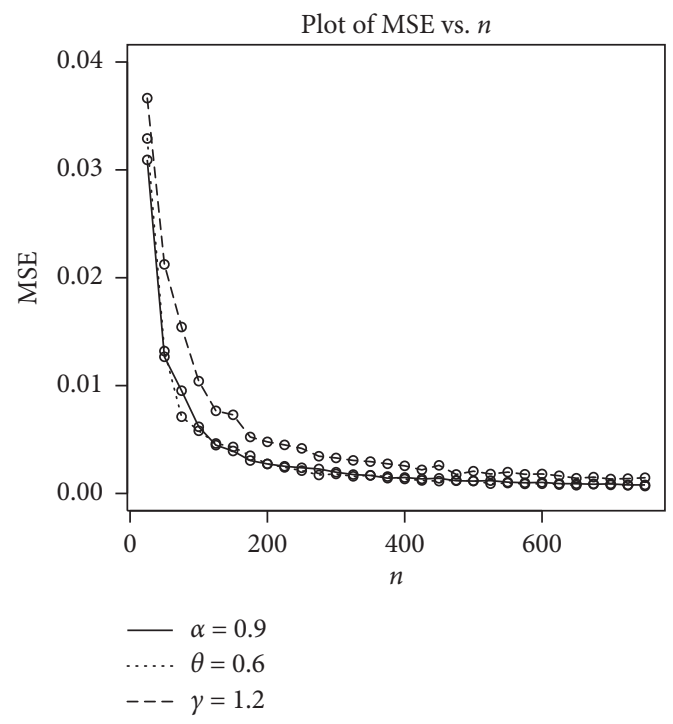

(b)

FIgURE 9: MLEs and MSEs plots of the NGE-Weibull distribution for $\alpha=0.9, \theta=0.6$, and $\gamma=1.2$.

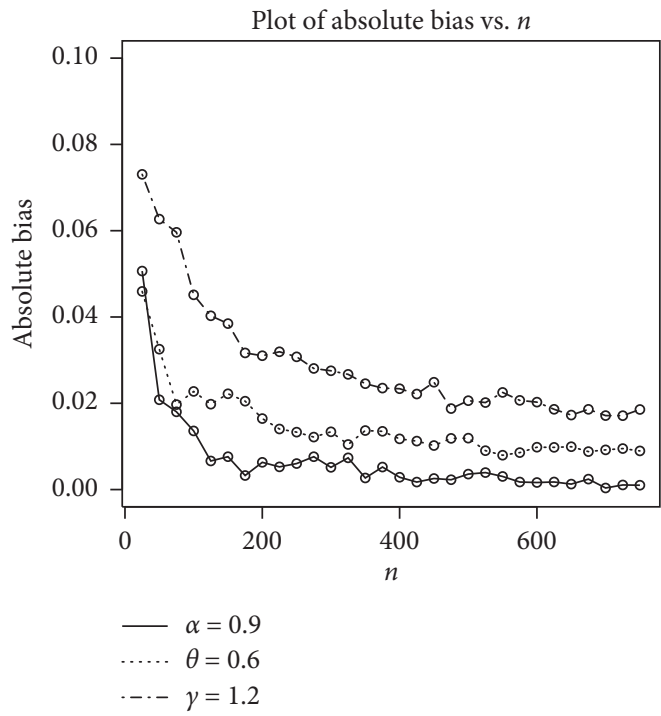

(a)

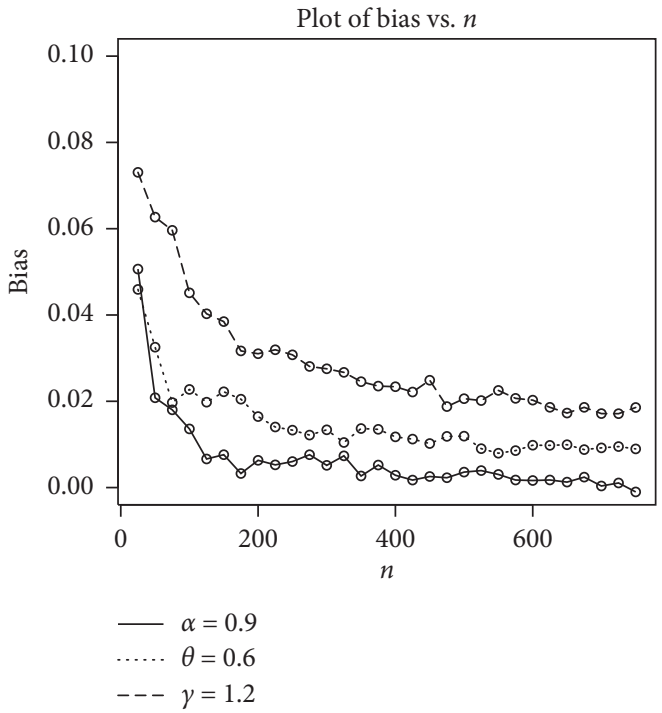

(b)

FIGURE 10: Biases and absolute biases plots of the NGE-Weibull distribution for $\alpha=0.9, \theta=0.6$, and $\gamma=1.2$. as

Thus, the Renyi entropy for $\delta>0, \delta \neq 1$, can be expressed

$$
\frac{1}{1-\delta} \log \left\{\sum_{k=0}^{\infty} \sum_{q=0}^{\infty} \sum_{m=0}^{\delta} \Omega_{k, q, m} \int_{-\infty}^{\infty} f(x)^{\delta} F(x)^{\delta+2(k+q+m)} \mathrm{d} x\right\} .
$$

3.8. Characterization Theorem. It is known that the failure rate function, $h_{F}$, of a twice differentiable function, $F$, satisfies the first-order differential equation:

$$
\frac{f^{\prime}(x)}{f(x)}=\frac{h_{F}^{\prime}(x)}{h_{F}(x)}-h_{F}(x) .
$$

In this section, we present a Weibull-NGE-X distribution. The result here is inspired by Alizadeh et al. [12]. First, let us introduce the following.

Definition 1 . We say a random variable $X$ follows a Weibull$G$ model if its cdf is given by

$$
F(x ; \xi)=1-e^{-\left(G(x ; \xi) / \overline{G(x ; \xi))^{\alpha}},\right.}
$$


where $G$ is some baseline distribution, $x \in \operatorname{Supp}(G)$, and $\xi$ is a vector of parameters in the baseline distribution whose support depends on $G, \alpha>0$, and $\bar{G}=1-G$.

The pdf of the Weibull- $G$ model is given by

$$
f(x ; \xi)=\alpha g(x ; \xi) \frac{G(x ; \xi)^{\alpha-1}}{\overline{G(x ; \xi)})^{\alpha+1}} e^{-\left(G(x ; \xi) / \overline{G(x ; \xi))^{\alpha}}\right.},
$$

where $g$ is the pdf of the baseline distribution. Clearly, the hazard rate function of the Weibull-G distribution is given by

$$
h_{F}(x ; \xi)=\alpha g(x ; \xi) \frac{G(x ; \xi)^{\alpha-1}}{\overline{G(x ; \xi)}} .
$$

Theorem 1. Let $X: \Omega \mapsto \mathbb{R}$ be a continuous random variable. The $p d f$ of $X$ is

$$
\alpha g(x ; \xi) \frac{G(x ; \xi)^{\alpha-1}}{\overline{G(x ; \xi)}} e^{-\left(G(x ; \xi) / \overline{G(x ; \xi))^{\alpha}},\right.}
$$

for some baseline distribution with $p d f g, c d f G, \alpha>0$, and $\bar{G}=1-G$, if and only if its hazard rate function $h_{F}(x)$ satisfies the differential equation given by

$$
h_{F}^{\prime}(x)-g^{\prime}(x) g(x)^{-1} h_{F}(x)=\alpha g(x) \frac{\mathrm{d}}{\mathrm{d} x} \frac{G(x)^{\alpha-1}}{\overline{G(x)}},
$$

with $x \in \mathbb{R}$, with the initial condition $h_{F}(0)=0$ for $\alpha>1$.

Proof. If $X$ has pdf as stated in the theorem, then the differential equation as stated in the theorem holds. Now if the stated differential equation holds, then

$$
\frac{\mathrm{d}}{\mathrm{d} x}\left\{g(x)^{-1} h_{F}(x)\right\}=\alpha \frac{\mathrm{d}}{\mathrm{d} x} \frac{G(x)^{\alpha-1}}{\overline{G(x)^{\alpha+1}}},
$$

or

$$
h_{F}(x ; \xi)=\alpha g(x ; \xi) \frac{G(x ; \xi)^{\alpha-1}}{\overline{G(x ; \xi)}},
$$

which is the hazard function of Weibull-G

Clearly, a characterization of the Weibull-NGE- $X$ distribution is obtained from the above theorem by letting the baseline pdf and cdf be given as in Section 1 .

\section{Estimation of the Parameters and Monte Carlo Simulation}

The section deals with the derivation of the MLEs (maximum likelihood estimators) of distribution of the NGEtextit $X$ and then conducts a simulation study using the Monte Carlo approach to evaluate the MLEs.

4.1. Maximum Likelihood Estimation. Consider a set of observed values, say $x_{1}, x_{2}, \ldots, x_{n}$, observed from the NGE$X$ distributions with parameters $\alpha$ and $\xi$. The total LLF (loglikelihood function) for $\Theta=(\alpha, \xi)$ is

$$
\begin{aligned}
\ell(\Theta)= & n \log 2+n \log \theta+\sum_{i=1}^{n} \log f(x ; \xi)+\sum_{i=1}^{n} \log F(x ; \xi) \\
& +(\theta-1) \sum_{i=1}^{n} \log \left(1-F(x ; \xi)^{2}\right) \\
& +\sum_{i=1}^{n} \log \left(2-F(x ; \xi)^{2}\right)-\theta \sum_{i=1}^{n} F(x ; \xi)^{2} .
\end{aligned}
$$

The partial derivatives of the LLF are given by

$$
\frac{\partial}{\partial \theta} \ell(\Theta)=\frac{n}{\theta}+\sum_{i=1}^{n} \log \left(1-F(x ; \xi)^{2}\right)-\theta \sum_{i=1}^{n} F(x ; \xi)^{2},
$$

and

$$
\begin{aligned}
\frac{\partial}{\partial \xi} \ell(\Theta)= & \sum_{i=1}^{n} \frac{\partial f(x ; \xi) / \partial \xi}{f(x ; \xi)}+\sum_{i=1}^{n} \frac{\partial F(x ; \xi) / \partial \xi}{F(x ; \xi)} \\
& -(\theta-1) \sum_{i=1}^{n} \frac{\partial F(x ; \xi)^{2} / \partial \xi}{\left(1-F(x ; \xi)^{2}\right)}-\sum_{i=1}^{n} \frac{\partial F(x ; \xi)^{2} / \partial \xi}{\left(2-F(x ; \xi)^{2}\right)} \\
& -\theta \sum_{i=1}^{n} \partial F(x ; \xi)^{2} \partial \xi .
\end{aligned}
$$

Setting these equations to zero and solving them simultaneously yields the MLEs of $\theta$ and $\xi$.

4.2. Monte Carlo Simulation Study. In this subsection, the MLEs of the NGE-Weibull distribution are evaluated via the Monte Carlo simulation approach. Measures such as mean square error (MSE), biases, and absolute biases are used for evaluation purposes. We generate $N=750$ of samples size $n=25,50, \ldots, 750$ from the proposed NGE-Weibull model using the inverse transformed technique. For each generated sample, MLEs $(\widehat{\alpha}, \widehat{\theta}, \widehat{\gamma})$ of the NGE-Weibull are obtained. The estimated biases and MSEs are calculated via the formulas given by

$$
\begin{aligned}
& \operatorname{Bias}_{\varepsilon}(n)=\frac{1}{N} \sum_{i=1}^{N}\left(\widehat{\varepsilon}_{i}-\varepsilon\right), \\
& \operatorname{MSE}_{\varepsilon}(n)=\frac{1}{N} \sum_{i=1}^{N}\left(\widehat{\varepsilon}_{i}-\varepsilon\right)^{2},
\end{aligned}
$$

respectively.

Figures 5-10 illustrate the simulation results for the above measures. These plots show that increasing sample size $n$ results in decreasing the estimated biases. Also, increasing sample size $n$ results in decreasing the estimated MSEs decay toward zero as $n$ increases. These results reveal the efficiency as well as the consistency property of the MLEs. 


\section{Data Analysis}

This section is devoted to illustrating the NGE-Weibull distribution by analyzing an application taken from reliability engineering. The data set representing the failure times of 50 electronic components (per 1000h) is given by $0.036,0.058,0.061,0.074,0.078,0.086,0.102,0.103,0.114$, $0.116,0.148,0.183,0.192,0.254,0.262,0.379,0.381,0.538$, $0.570,0.574,0.590,0.618,0.645,0.961,1.228,1.600,2.006$, $2.054,2.804,3.058,3.076,3.147,3.625,3.704,3.931,4.073$, $4.393,4.534,4.893,6.274,6.816,7.896,7.904,8.022,9.337$, $10.940,11.020,13.880,14.730,15.080$. For more details about this data, see Aryal and Elbatal [13].

The MLEs of the NGE-Weibull and other competing distributions are determined, and seven analytical measures including three goodness-of-fit statistics such as Cramer-Von Mises (CM) test statistic, Anderson Darling (AD) test statistic, and Kolmogorov-Smirnov (KS) statistic along with $p$-value and four discrimination measures such as Akaike information criterion (AIC), Consistent Akaike Information Criterion (CAIC), Bayesian information criterion (BIC), and Hannan-Quinn information criterion (HQIC) are considered. For more details about these measures, we refer to Ahmad et al. [7]. All the computations have been carried via the optim() Rfunction with the argument method= "BFGS"; see Appendix.

In general, a model with the smaller values of these statistics represents the better fit to data. We fit the proposed model and other models to the failure time data set. The other fitted models are: the two-parameter Weibull and three-parameter exponentiated Weibull (EW) of Modhalkar and Sarivasta [14] and Marshall-Olkin Weibull (MOW) of the Marshall and Olkin [15]. The pdfs of the competing models are as follows:

(i) Weibull distribution:

$$
f(x)=\alpha \gamma x^{\alpha-1} e^{-\gamma x^{\alpha}}, \quad x, \alpha, \gamma>0
$$

(ii) EW distribution:

$$
f(x)=\alpha \gamma \theta x^{\alpha-1} e^{-\gamma x^{\alpha}}\left(1-e^{-\gamma x^{\alpha}}\right)^{\theta-1}, \quad x, \alpha, \gamma, \theta>0 .
$$

(iii) MOW distribution:

$$
f(x)=\frac{\alpha \gamma \sigma x^{\alpha-1} e^{-\gamma x^{\alpha}}}{\sigma+(1-\sigma)\left(1-e^{-\gamma x^{\alpha}}\right)}, \quad x, \alpha, \gamma, \sigma>0 .
$$

The MLEs and their corresponding standard errors (in parentheses) of the model parameters are presented in Table 3. The discrimination measures of all the competing models are presented in Table 4 whereas the goodness-of-fit measures are reported in Table 5.

The results of the NGE-Weibull are compared with the Weibull, EW, and MOW models in Tables 4 and 5. From these results, we see that NGE-Weibull has the lowest values for the considered measures. Therefore, we conclude that the NGE-Weibull model can be selected as the best competitive model for data related to the failure times of the electronic devices.

In addition to the numerical results provided in Tables 4 and 5, the plots of the fitted density and distribution function of the NGE-Weibull model are displayed in Figure 11. The Kaplan-Meier survival and the probability-probability (PP) are shown in Figure 12. From the results provided in Tables 4 and 5 and displayed graphically in Figures 11 and 12, we see that the NGE-Weibull provides a close fit to the reliability engineering data.

In addition, for the failure times of electronic components, we calculate the KS statistical values of the NGEWeibull distribution and other competitors. Subsequently, we applied the parametric bootstrap method [16] and bootstrapped the $p$ value for all distributions. The KS statistic and the corresponding bootstrapped $p$ value are reported in Table 6 . Based on the results presented in Table 6, we see that NGE-Weibull is a good competitor among the competing models for modeling the failure times of the electronic component's data.

\section{Bayesian Estimation}

The Bayesian inference has been taken into consideration by a number of researchers. In the Bayesian analysis, we do not know the exact value of the model parameters, which can be negatively affected by the loss when selecting an estimator. These losses can be measured by the function of the parameter and the corresponding estimator. Here, in the Bayesian analysis, we consider different types of symmetric and asymmetric loss functions; see Table 7.

For further details, we refer to Kharazmi et al. [17] and Ahmad et al. [7].

Next, we provide a Bayesian discussion for estimating the parameters of NGE-Weibull distribution via analyzing complete sample data.

6.1. Joint Posterior and Marginal Posterior Distributions. Assume that the parameters $\alpha, \gamma$, and $\theta$ of the NGE-Weibull have independent prior distributions defined by

$$
\alpha \sim \Gamma\left(\alpha_{0}, \alpha_{1}\right), \gamma \sim \Gamma\left(\gamma_{0}, \gamma_{1}\right), \theta \sim \Gamma\left(\theta_{0}, \theta_{1}\right),
$$

where $\alpha_{0}, \alpha_{1}, \gamma_{0}, \gamma_{1}, \theta_{0}, \theta_{1}>0$. Consequently, the joint prior density function can be formulated as follows:

$$
\pi(\alpha, \gamma, \theta)=\frac{\alpha_{1}^{\alpha_{0}} \gamma_{1}^{\gamma_{0}} \theta_{1}^{\theta_{0}}}{\Gamma\left(\alpha_{0}\right) \Gamma\left(\gamma_{0}\right) \Gamma\left(\theta_{0}\right)} \alpha^{\alpha_{0}-1} \gamma^{\gamma_{0}-1} \theta^{\theta_{0}-1} e^{-\left(\alpha_{1} \alpha+\gamma_{1} \gamma+\theta_{1} \theta\right)} .
$$

For simplicity, let us define the function $\zeta$ as

$$
\zeta(\alpha, \gamma, \theta)=\alpha^{\alpha_{0}-1} \gamma^{\gamma_{0}-1} \theta^{\theta_{0}-1} e^{-\left(\alpha_{1} \alpha+\gamma_{1} \gamma+\theta_{1} \theta\right)}, \quad \alpha>0, \gamma>0, \theta>0 .
$$

The joint posterior distribution defined from equation (64) and the likelihood function $L$ (data) is 
TABLE 3: The MLEs with standard errors of the competing models for the failure time data.

\begin{tabular}{lcccc}
\hline Dist. & $\widehat{\alpha}$ & $\widehat{\gamma}$ & $\widehat{\theta}$ & $\widehat{\sigma}$ \\
\hline NGE-Weibull & $0.657(0.0735)$ & $4.251(1.5272)$ & $0.125(0.0550)$ & - \\
Weibull & $0.661(0.0747)$ & $0.541(0.0994)$ & - & - \\
EW & $0.740(0.5262)$ & $0.419(0.6802)$ & $0.824(0.9068)$ & - \\
MOW & $0.727(0.1395)$ & $0.380(0.2614)$ & - & $0.604(0.5531)$ \\
\hline
\end{tabular}

TABLE 4: The discrimination measures of the competing models for the failure time data.

\begin{tabular}{lcccc}
\hline Dist. & AIC & BIC & CAIC & HQIC \\
\hline NGE-Weibull & 206.168 & 211.904 & 206.690 & 208.353 \\
Weibull & 208.728 & 212.552 & 208.984 & 210.184 \\
EW & 210.716 & 216.452 & 211.238 & 212.901 \\
MOW & 210.432 & 216.168 & 210.954 & 212.617 \\
\hline
\end{tabular}

TABLE 5: The analytical measures of the competing models for the failure time data.

\begin{tabular}{lcccc}
\hline Dist. & CM & AD & KS & $p$ value \\
\hline NGE-Weibull & 0.121 & 0.758 & 0.110 & 0.535 \\
Weibull & 0.152 & 0.954 & 0.126 & 0.365 \\
EW & 0.150 & 0.947 & 0.129 & 0.344 \\
MOW & 0.152 & 0.952 & 0.114 & 0.491 \\
\hline
\end{tabular}
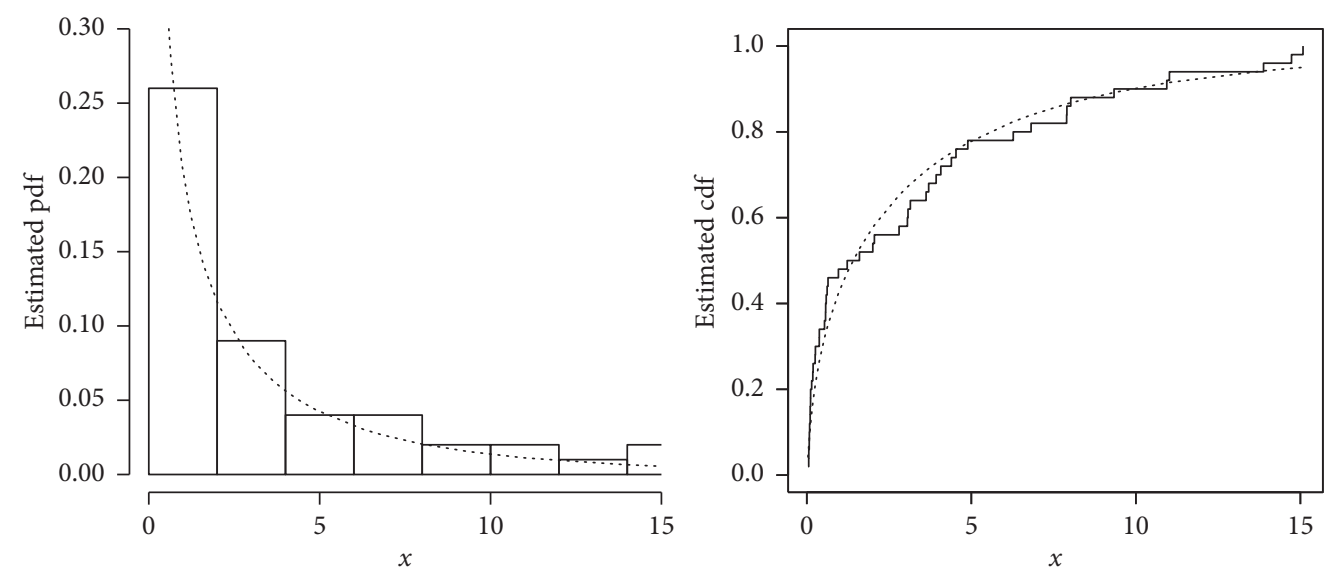

Figure 11: Plots of fitted pdf and cdf of the NGE-Weibull distribution.
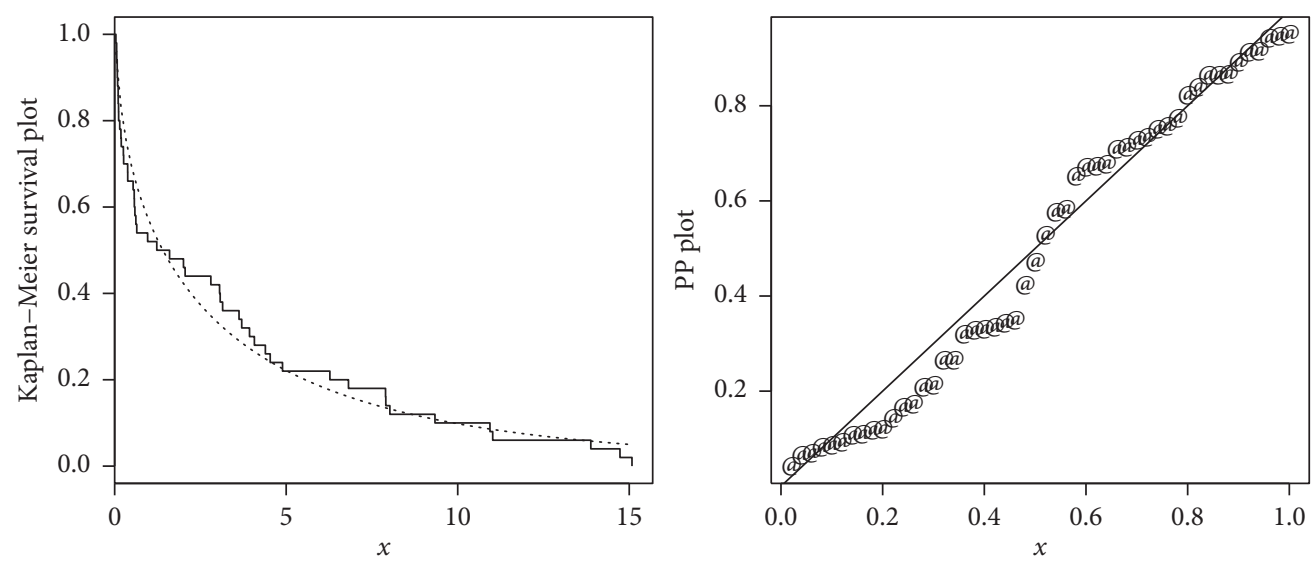

Figure 12: Plots of the PP and the Kaplan-Meier survival function of the NGE-Weibull distribution. 
TABLE 6: The KS and the corresponding bootstrapped $p$ value of the fitted models for the failure times of electronic components.

\begin{tabular}{lcr}
\hline Distributions & KS & Bootstapped $p$ value \\
\hline NGE-Weibull & 0.150 & 0.850 \\
Weibull & 0.420 & 0.549 \\
EW & 0.479 & 0.504 \\
MOW & 0.376 & 0.694 \\
\hline
\end{tabular}

TABLe 7: Bayes estimator and posterior risk under different loss functions.

\begin{tabular}{lcr}
\hline Loss function $L(\psi, \delta)$ & Bayes estimator $\psi_{B}$ & Posterior risk $\rho_{\psi}$ \\
\hline SELF $=(\psi-d)^{2}$ & $E(\psi \mid x)$ & $\operatorname{Var}(\psi \mid x)$ \\
WSELF $=\left((\psi-d)^{2} / \psi\right)$ & $\left(E\left(\psi^{-1} \mid x\right)\right)^{-1}$ & $E(\psi \mid x)-\left(E\left(\psi^{-1} \mid x\right)\right)^{-1}$ \\
MSELF $=(1-(d / \psi))^{2}$ & $\left(E\left(\psi^{-1} \mid x\right) / E\left(\psi^{-2} \mid x\right)\right)$ & $1-\left(E\left(\psi^{-1} \mid x\right)^{2} / E\left(\psi^{-2} \mid x\right)\right)$ \\
PLF $=\left((\psi-d)^{2} / d\right)$ & $\sqrt{E\left(\psi^{2} \mid x\right)}$ & $2\left(\sqrt{E\left(\psi^{2} \mid x\right)}-E(\psi \mid x)\right)$ \\
$\operatorname{KLF}=(\sqrt{(d / \psi)-\sqrt{(\psi / d)}})$ & $\sqrt{\left(E(\psi \mid x) / E\left(\psi^{-1} \mid x\right)\right)}$ & $2\left(\sqrt{E(\psi \mid x) E\left(\psi^{-1} \mid x\right)}-1\right)$ \\
\hline
\end{tabular}

TABLE 8: Bayesian estimates and their posterior risks of the parameters under different loss functions based on the failure time data.

\begin{tabular}{|c|c|c|c|c|c|c|}
\hline \multirow{3}{*}{$\begin{array}{l}\text { Data } \\
\text { Bayes } \\
\text { Loss functions }\end{array}$} & \multicolumn{6}{|c|}{ Insurance data } \\
\hline & \multicolumn{2}{|c|}{$\widehat{\alpha}$} & \multicolumn{2}{|c|}{$\widehat{\gamma}$} & \multicolumn{2}{|c|}{$\widehat{\theta}$} \\
\hline & Estimate & Risk & Estimate & Risk & Estimate & Risk \\
\hline SELF & 0.2280 & 0.0007 & 2.2772 & 0.2107 & 0.1560 & 0.0007 \\
\hline WSELF & 0.2249 & 0.0031 & 2.1881 & 0.0891 & 0.1516 & 0.0044 \\
\hline MSELF & 0.2218 & 0.0138 & 2.1025 & 0.0391 & 0.1472 & 0.0292 \\
\hline PLF & 0.2295 & 0.0030 & 2.3230 & 0.0916 & 0.1582 & 0.0044 \\
\hline KLF & 0.2265 & 0.0136 & 2.2322 & 0.0403 & 0.1538 & 0.0291 \\
\hline
\end{tabular}

TABLE 9: Credible and HPD intervals of the parameters $\alpha, \gamma$, and $\theta$ for the failure time data.

\begin{tabular}{lcr}
\hline Parameters & Credible interval & HPD interval \\
\hline$\alpha$ & $(0.2096,0.2451)$ & $(0.1794,0.2819)$ \\
$\gamma$ & $(1.9490,2.5563)$ & $(1.4270,3.1600)$ \\
$\theta$ & $(0.1377,0.1725)$ & $(0.1082,0.2101)$ \\
\hline
\end{tabular}

$$
\pi^{*}(\alpha, \gamma, \theta \mid \text { data }) \propto \pi(\alpha, \gamma, \theta) L(\text { data }) .
$$

Therefore, the joint posterior pdf can be expressed by

$$
\pi^{*}(\alpha, \gamma, \theta \mid \underline{x})=K \zeta(\alpha, \gamma, \theta) L(\underline{x}, \Psi),
$$

where

$$
L(\underline{x} ; \Psi)=(2 \alpha \gamma \theta)^{n} \prod_{i=1}^{n} \frac{x_{i}^{\alpha-1} e^{-\left\{\theta\left(1-e^{-\gamma x_{i}^{\alpha}}\right)^{2}+\gamma x_{i}^{\alpha}\right\}}\left(1-e^{-\gamma x_{i}^{\alpha}}\right)}{\left.\left(1-e^{-\gamma x_{i}^{\alpha}}\right)^{2}\right)^{1-\theta}\left(2-\left(1-e^{-\gamma x_{i}^{\alpha}}\right)^{2}\right)},
$$

$\Psi=(\alpha, \gamma, \theta)$, and $K$ is given as

$$
K^{-1}=\int_{0}^{\infty} \int_{0}^{\infty} \int_{0}^{\infty} \zeta(\alpha, \gamma, \theta) L(\underline{x}, \Psi) \partial \alpha \partial \gamma \partial \theta .
$$

Moreover, the marginal posterior pdf of $\alpha, \gamma$, and $\theta$ assuming that $\Psi=\left(\Psi_{1}, \Psi_{2}, \Psi_{3}\right)=(\alpha, \gamma, \theta)$, can be given as

$$
\pi\left(\Psi_{i} \mid \underline{x}\right)=\int_{0}^{\infty} \int_{0}^{\infty} \pi^{*}(\Psi \mid \underline{x}) \partial \Psi_{j} \partial \Psi_{k}
$$

where $i, j, k=1,2,3, i \neq j \neq k$, and $\Psi_{i}$ is the $i$ th member of a vector $\Psi$.
6.2. Bayesian Point Estimation. Under the marginal posterior pdf as in (70) and the loss functions which are given in Table 7, the Bayesian point estimation for the parameter vector $\Psi=\left(\Psi_{1}, \Psi_{2}, \Psi_{3}\right)=(\alpha, \gamma, \theta)$ is obtained via minimizing the expectation of loss function under the marginal posterior pdf as follows:

$$
\operatorname{argmin}_{\delta} \int_{0}^{\infty} L\left(\Psi_{i}, \delta\right) \pi\left(\Psi_{j} \mid \underline{x}\right) \partial \Psi_{i}
$$

However, in practice, because of the intractable integral in relation (71), using the well-known Gibbs sampler [18] or Metropolis Hastings algorithms $[19,20]$ is suggested to generate posterior samples. We will argue this issue more precisely in subsection 6.5 .

6.3. Credibility Interval. In the Bayesian framework, interval estimation is done via credibility interval conception. Consider the parameter vector $\Psi=\left(\Psi_{1}, \Psi_{2}, \Psi_{3}\right)=$ $(\alpha, \gamma, \theta)$, which is associated with the NGE-Weibull distribution and $\pi\left(\Psi_{i} \mid \underline{x}\right)$ denote the marginal posterior pdf of the parameter $\Psi_{j} ;(j=1,2,3)$ as in (70). For a given value of $\eta \in(0,1)$, the $(1-\eta) 100 \%$ credibility interval $\mathrm{CI}\left(L_{\Psi_{j}}, U_{\Psi_{j}}\right)$ is defined as 


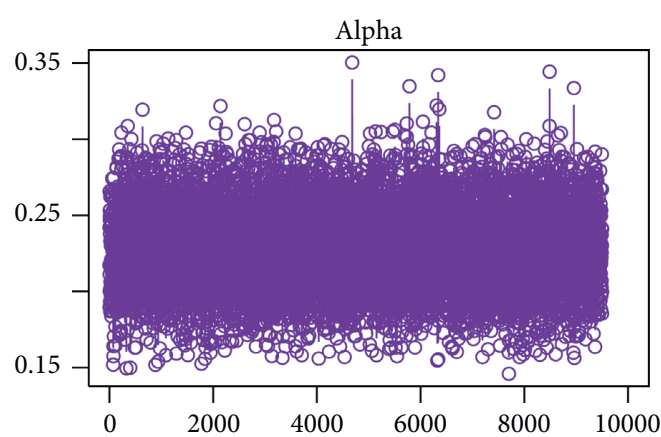

(a)

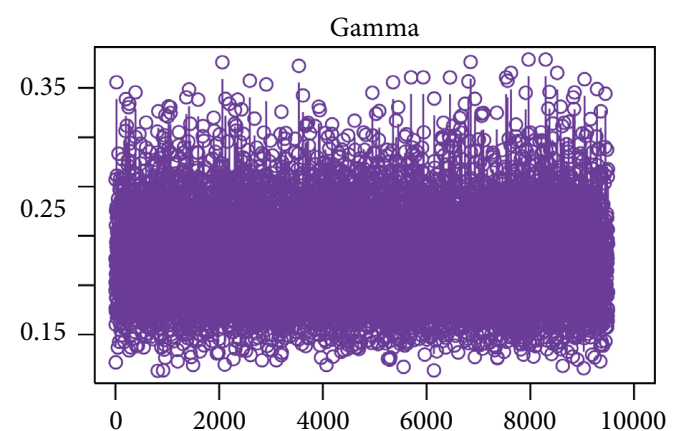

(b)

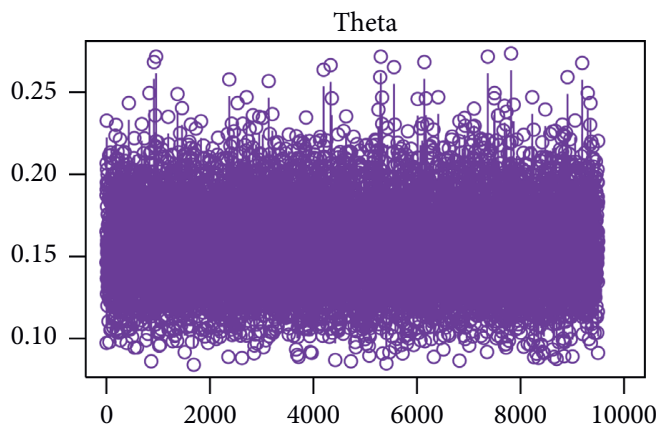

(c)

Figure 13: Trace plots of each parameter of NGE-Weibull distribution.

$$
\begin{aligned}
& \int_{L_{\Psi_{j}}}^{\infty} \pi\left(\Psi_{j} \mid \underline{x}\right) \partial \Psi_{j}=1-\frac{\eta}{2}, \\
& \int_{U_{\Psi_{j}}}^{\infty} \pi\left(\Psi_{j} \mid \underline{x}\right) \partial \Psi_{i}=\frac{\eta}{2} .
\end{aligned}
$$

By considering the relation (72), it is very difficult to obtain the marginal pdf from the joint posterior pdf. We use the Gibbs sampler to generate posterior samples. Let $\Psi^{1}, \ldots, \Psi^{k}\left(\right.$ where $\left.\Psi^{i}=\left(\Psi_{1}^{i}, \Psi_{2}^{i}, \Psi_{3}^{i}\right)\right)$ be a posterior random sample of size $k$, which is extracted from the joint posterior pdf as in (67). Using these generated posterior samples, the marginal posteriors pdfs of $\Psi_{j}$ given $\underline{x}$ can be given by

$$
\frac{1}{K} \sum_{i=1}^{K} \pi^{*}\left(\Psi_{j}, \Psi_{-j}^{i} \mid \underline{x}\right) ; \quad j=1,2,3,
$$

where $\Psi_{-j}^{i}$ shows the vector of posterior samples when the $j^{\text {th }}$ component is removed. Using (73) in (72), one can be able to compute the credibility intervals for $\Psi_{j}, j=1,2,3$ as follows:

$$
\begin{aligned}
& \frac{1}{K} \sum_{i=1}^{K} \int_{L_{\Psi_{j}}}^{\infty} \pi^{*}\left(\Psi_{j}, \Psi_{-j}^{i} \mid \underline{x}\right) \partial \Psi_{j}=1-\frac{\eta}{2}, \\
& \frac{1}{K} \sum_{i=1}^{K} \int_{U_{\Psi_{j}}}^{\infty} \pi^{*}\left(\Psi_{j}, \Psi_{-j}^{i} \mid \underline{x}\right) \partial \Psi_{j}=\frac{\eta}{2} .
\end{aligned}
$$

6.4. The Highest Posterior Density Interval. The highest posterior density interval is a kind of credibility interval which imposed a specific restriction. A $(1-\eta) 100 \%(i=$
$1, \ldots, p$ ) HPD interval for $\Psi_{j}, j=1,2,3$, is the simultaneous solution of the following integral equations:

$$
\begin{aligned}
& \frac{1}{K} \sum_{i=1}^{K} \int_{L_{\Psi_{j}}}^{U_{\Psi_{j}}} \pi^{*}\left(\Psi_{j}, \Psi_{-j}^{i} \mid \underline{x}\right) \partial \Psi_{j}=1-\eta \\
& \sum_{i=1}^{K} \pi^{*}\left(L_{\Psi_{j}}, \Psi_{-j}^{i} \mid \underline{x}\right)=\sum_{i=1}^{K} \pi^{*}\left(U_{\Psi_{j}}, \Psi_{-j}^{i} \mid \underline{x}\right) .
\end{aligned}
$$

6.5. Generating Posterior Samples. It is clear from (67) and (70) that there are no explicit expressions for the Bayesian point estimators under the loss functions; see Table 7. Due to the intractable integrals associated with joint posterior and marginal posterior distributions, therefore, we require numerical software to solve numerically the integral equations via MCMC methods such as Gibbs sampling and the Metropolis-Hastings algorithm.

Suppose that the general model $f(\underline{x} \mid \psi)$ is associated with parameter vector $\psi=\left(\psi_{1}, \psi_{2}, \ldots, \psi_{p}\right)$ and observed data $\underline{x}$. Thus, the joint posterior distribution is $\pi\left(\psi_{1}, \psi_{2}, \ldots, \psi_{p} \mid \underline{x}\right)$. We also assume that $\psi_{0}=\left(\psi_{1}^{(0)}, \psi_{2}^{(0)}\right.$, $\left.\ldots, \psi_{p}^{(0)}\right)$ is the initial values vector to start the Gibbs sampler. The Gibbs sampling approach draws the values for each iteration in $p$ steps by drawing a new value for each parameter from its full conditional given the most recently drawn values of all other parameters. The steps for any iteration, say iteration $k$, are as follows:

(i) Starting with an initial estimate $\left(\psi_{1}^{(0)}, \psi_{2}^{(0)}, \ldots, \psi_{p}^{(0)}\right)$ 


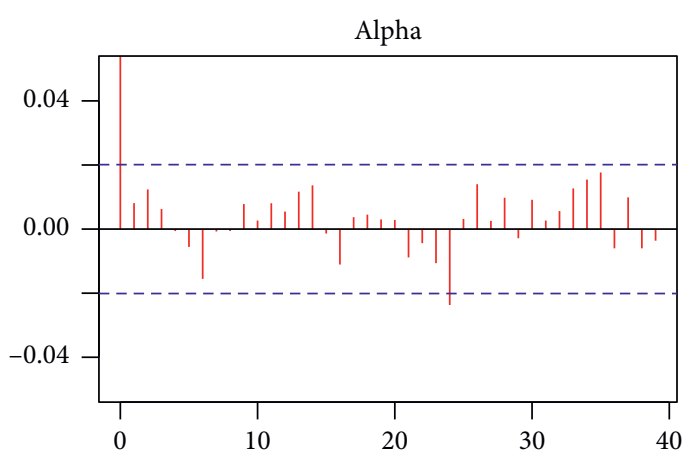

(a)

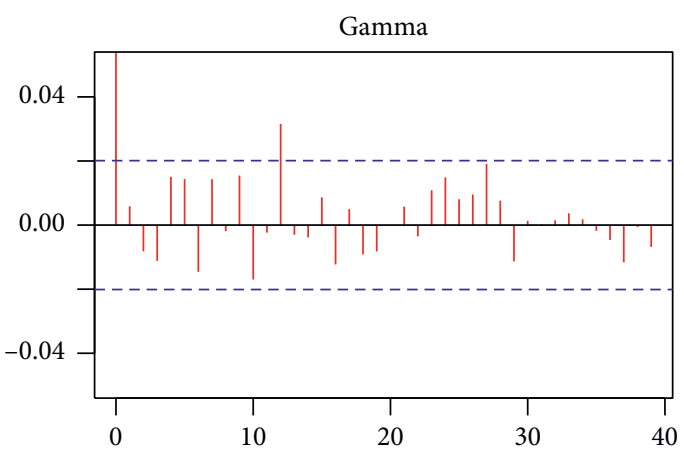

(b)

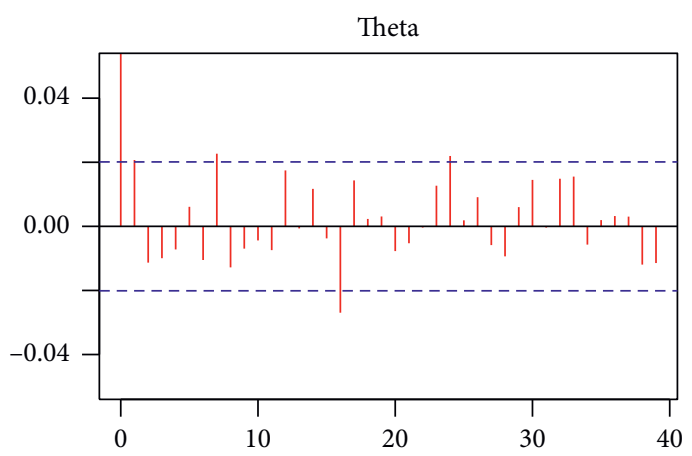

(c)

FIgURE 14: Autocorrelation plots of each parameter of NGE-Weibull distribution.

(ii) Draw $\psi_{1}^{k}$ from $\pi\left(\psi_{1} \mid \psi_{2}^{k-1}, \psi_{3}^{k-1}, \ldots, \psi_{p}^{k-1}, \underline{x}\right)$

(iii) Draw $\psi_{2}^{k}$ from $\pi\left(\psi_{2} \mid \psi_{1}^{k}, \psi_{3}^{k-1}, \ldots, \psi_{p}^{k-1}, \underline{x}\right)$ and so on down to

(iv) Draw $\psi_{p}^{k}$ from $\pi\left(\psi_{p} \mid \psi_{1}^{k}, \psi_{2}^{k}, \ldots, \psi_{p-1}^{k}, \underline{x}\right)$
In the case of the NGE-Weibull distribution, by considering the parameter vector $\Psi=(\alpha, \gamma, \theta)$ and initial parameter vector $\Psi_{0}=c\left(\alpha^{0}, \gamma^{0}, \theta^{0}\right)$, the posterior samples are extracted by the above Gibbs sampler where the full conditional distributions are given as

$$
\begin{aligned}
& \pi\left(\alpha \mid \gamma^{k-1}, \theta^{k-1}, \underline{x}\right) \propto \alpha^{\alpha_{0}+n} e^{-\alpha_{1} \alpha} \prod_{i=1}^{n} \frac{x_{i}^{\alpha-1} e^{-\left\{\theta\left(1-e^{-\gamma x_{i}^{\alpha}}\right)^{2}+\gamma x_{i}^{\alpha}\right\}}\left(1-e^{-\gamma x_{i}^{\alpha}}\right)}{\left(1-\left(1-e^{-\gamma x_{i}^{\alpha}}\right)^{2}\right)^{1-\theta}\left(2-\left(1-e^{-\gamma x_{i}^{\alpha}}\right)^{2}\right)}, \\
& \pi\left(\gamma \mid \alpha^{k-1}, \theta^{k-1}, \underline{x}\right) \propto \gamma^{\gamma_{0}+n} e^{-\gamma_{1} \gamma} \prod_{i=1}^{n} \frac{e^{-\left\{\theta\left(1-e^{-\gamma x_{i}^{\alpha}}\right)^{2}+\gamma x_{i}^{\alpha}\right\}}\left(1-e^{-\gamma x_{i}^{\alpha}}\right)}{\left(1-\left(1-e^{-\gamma x_{i}^{\alpha}}\right)^{2}\right)^{1-\theta}\left(2-\left(1-e^{-\gamma x_{i}^{\alpha}}\right)^{2}\right)}, \\
& \pi\left(\theta \mid \alpha^{k-1}, \gamma^{k-1}, \underline{x}\right) \propto \theta^{\theta_{0}+n} e^{-\theta_{1} \theta} \prod_{i=1}^{n} \frac{e^{-\theta\left(1-e^{-\gamma x_{i}^{\alpha}}\right)^{2}}}{\left(1-\left(1-e^{-\gamma x_{i}^{\alpha}}\right)^{2}\right)^{1-\theta} .}
\end{aligned}
$$

Here, since there is not any prior information about hyperparameters in (57), we implement the idea of Congdon [21] and the hyperparameters values are set as $\alpha_{0}=\alpha_{1}=\gamma_{0}=\gamma_{1}=\theta_{0}=\theta_{1}=0.0001$. So, we can use the MCMC procedure to extract posterior samples of (70) by means of the Gibbs sampling process in OpenBUGS software.
Next, we provide Bayesian estimation results. It is evident from equation (70) that there are no closed-form expressions for Bayesian estimators, which are extracted based on the loss functions in Table 7. Therefore, a MCMC procedure via the Gibbs sampler process is designed using the expressions (72), (73), and (74), with 10,000 replicates to obtain the Bayesian estimators. In Table 8 , we provide the 


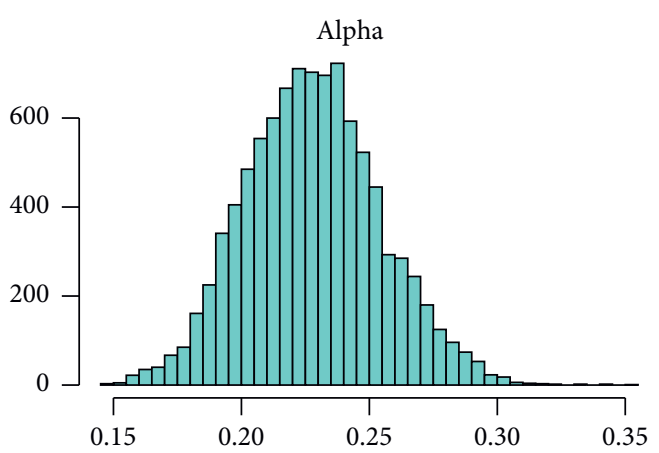

(a)

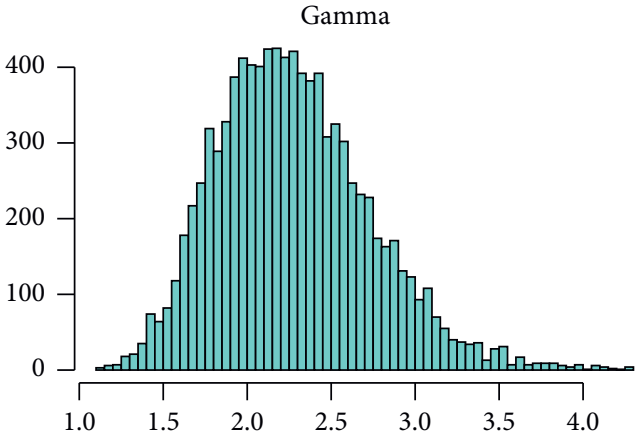

(b)

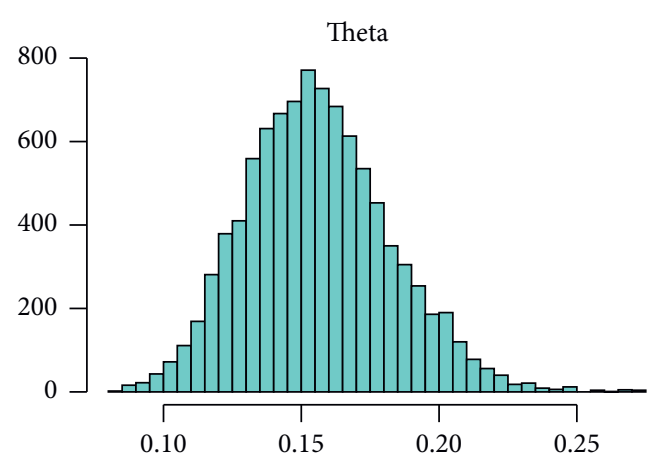

(c)

FIGURE 15: Histogram plots of each parameter of NGE-Weibull distribution.

TABLe 10: Diagnostics by the Gelman-Rubin, Geweke, and Raftery-Lewis methods for the parameters $\alpha, \gamma$, and $\theta$ based on the failure time data.

\begin{tabular}{lccc}
\hline Parameter & Gelman-Rubin & Geweke $\left(Z_{0.025}= \pm 1.96\right)$ & Raftery-Lewis \\
\hline$\alpha$ & 1 & 0.846 & 3.47 \\
$\gamma$ & 1 & 0.080 & 3.97 \\
$\theta$ & 1 & 1.727 & 4.5 \\
\hline
\end{tabular}

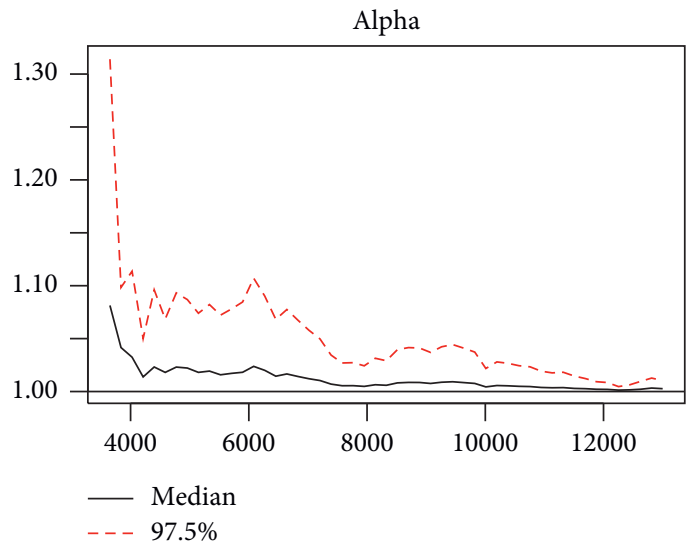

(a)

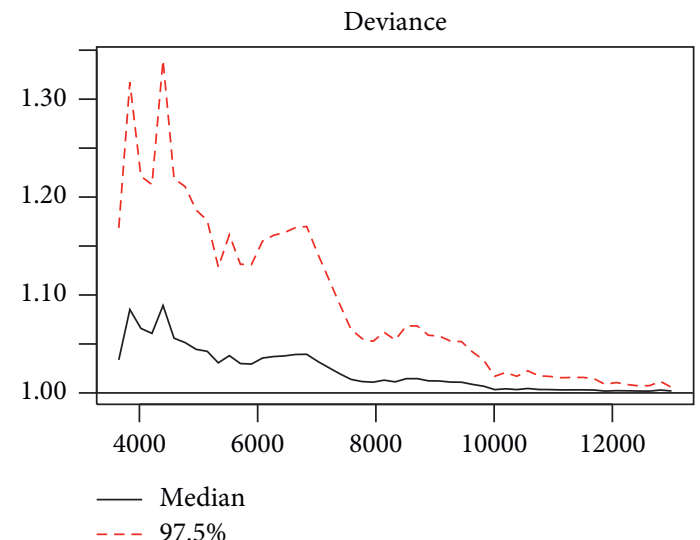

(b)

FIgure 16: Continued. 


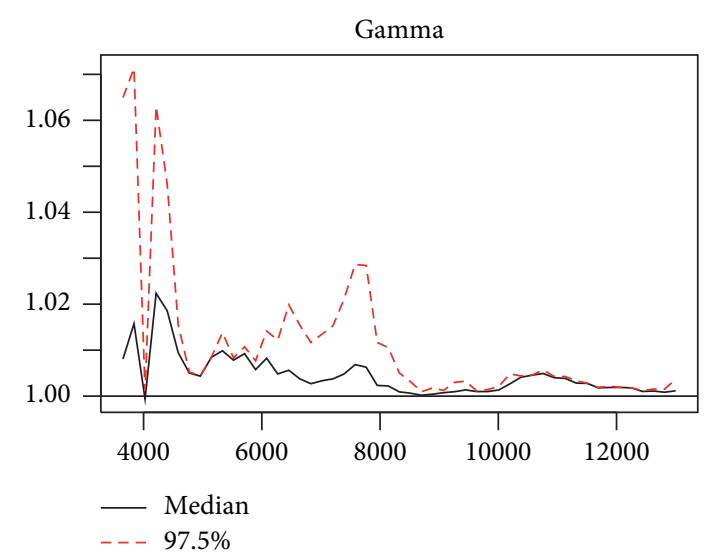

(c)

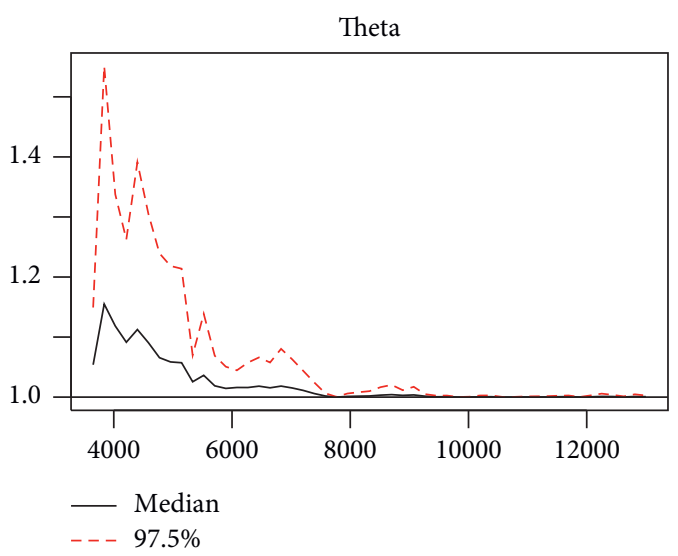

(d)

FIgURE 16: Gelman plot diagnostic for each parameter of NGE-Weibull distribution based on the failure time data.

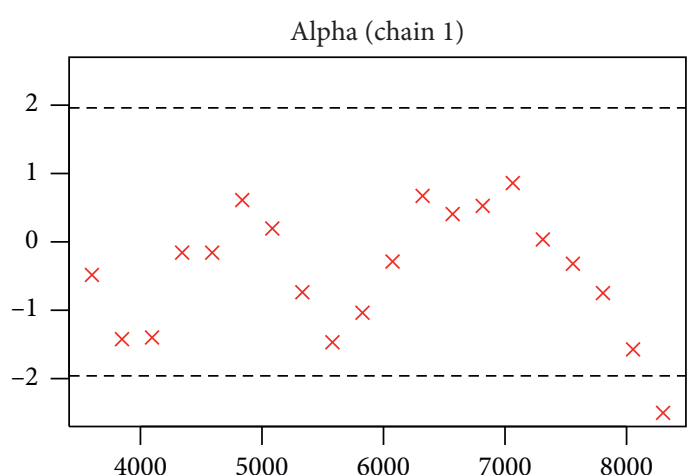

(a)

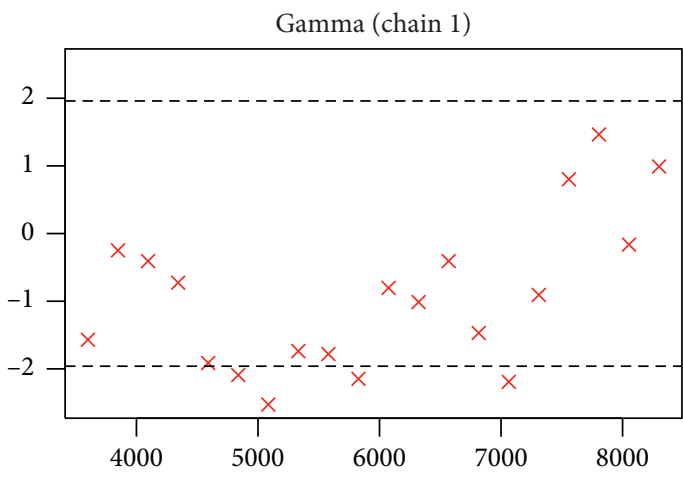

(c)

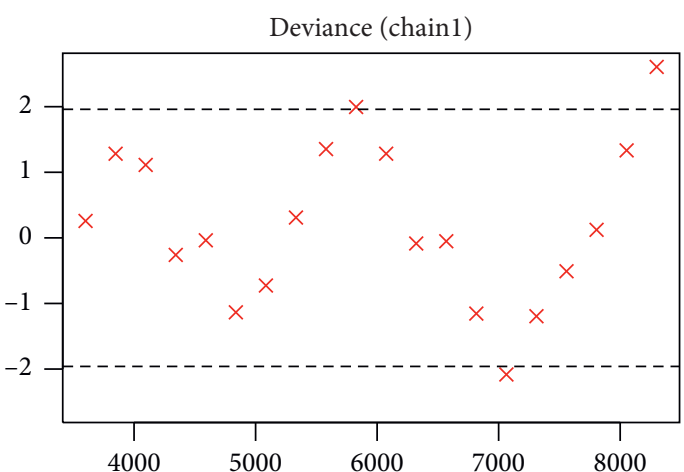

(b)

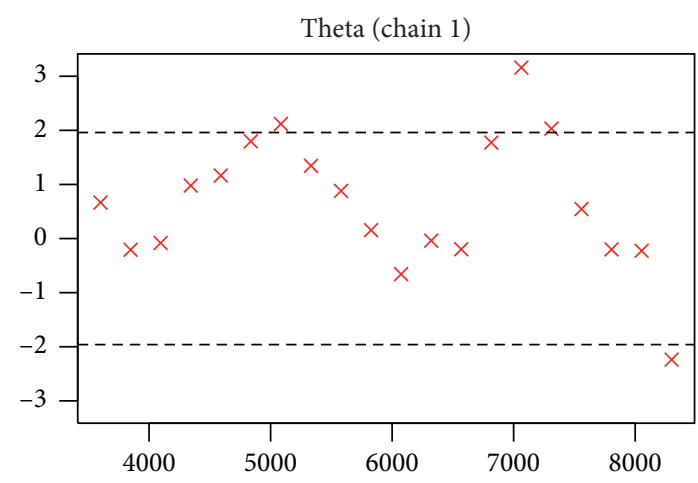

(d)

FIGURE 17: Geweke plot diagnostic (chain 1) for each parameter of NGE-Weibull distribution based on the failure time data.

corresponding point and posterior risk estimations. Furtherer, 95\% credible and HPD intervals are provided in Table 9. In order to provide a visual inspection, we provide posterior summary plots in Figures 13-15. These plots verify that the convergence of the Gibbs sampling process has occurred.
Next, for evaluation of the MCMC procedure in Bayesian analysis, we report some diagnostics measures such as Gelman-Rubin (GR), Geweke (G), and Raftery-Lewis (RL) for checking the convergence of the Gibbs algorithm in Table 10. For more details about these indexes, see Lee et al. [22]. The GR diagnostic for parameters $\alpha, \gamma$, and $\theta$ is equal to 


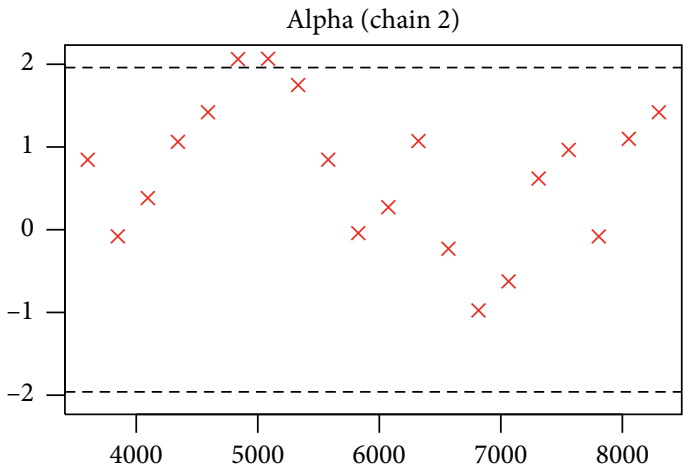

(a)

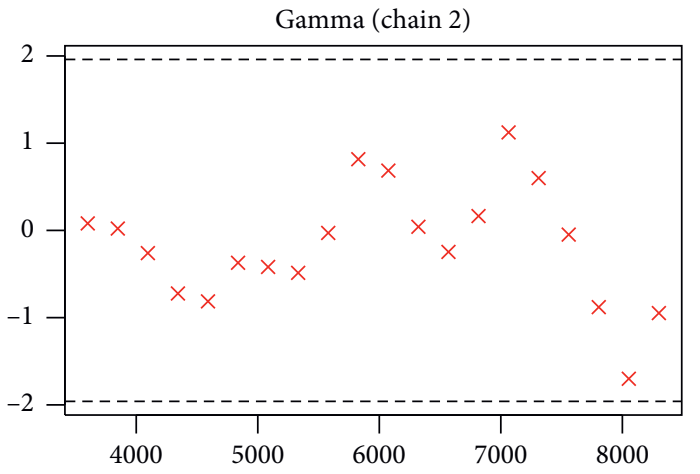

(c)

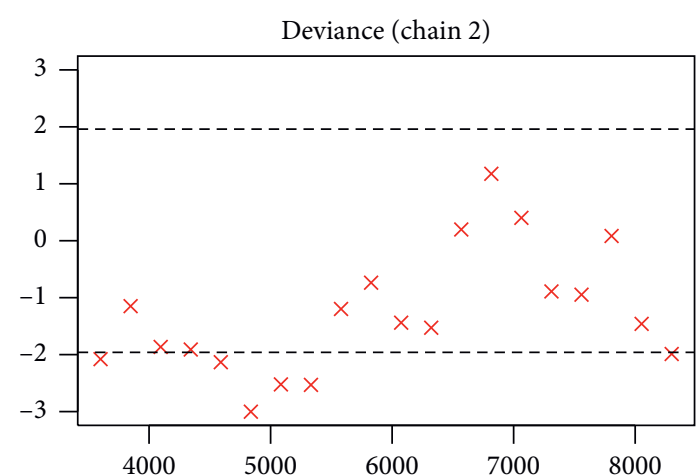

(b)

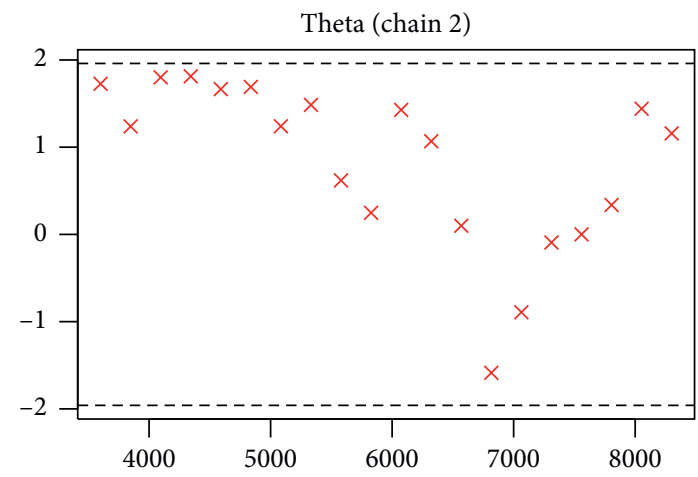

(d)

FIGURE 18: Geweke plot diagnostic (chain 2) for each parameter of NGE-Weibull distribution based on the failure time data.

1. Hence, based on the GR diagnostic measure, the chains are acceptable. Figure 16 shows that the estimates come from state spaces of the corresponding parameters. From Table 10, Geweke's test statistics for parameters $\alpha, \gamma$, and $\theta$ are 0.846 , 0.080 , and 1.727 , respectively. Hence, the $G$ diagnostic measure also confirms the acceptance of chains as shown in Figures 17 and 18. Moreover, the reported diagnostic statistics for parameters $\alpha, \gamma$, and $\theta$ based on the RL method do not show a significant degree of dependence between estimates.

\section{Concluding Remarks}

We have introduced a new generalized exponential- $X$ family. A special submodel of this family named a new generalized exponential-Weibull distribution is discussed. The density of the NGE-Weibull model can take different shapes of density and failure rate functions. Parameters of the NGE-Weibull distribution are estimated using the method of maximum likelihood estimation. A simulation study was conducted to evaluate the behavior of the estimators. Statistical properties of the NGE- $X$ distributions are also obtained. A real application related to the failure times data is considered and it is observed that the NGEWeibull model provides the best fit to data than other wellknown competitors. Finally, the Bayesian estimation method is used to estimate the model parameters and conduct the Bayesian analysis under five different loss functions. Furthermore, the diagnostics measures such as the Gelman-Rubin, Geweke, and Raftery Lewis are also discussed to evaluate the MCMC procedure in the Bayesian analysis.

\section{Appendix}

\section{A. R Code for Analysis}

Note: in the following R-code, pm is used for the proposed model.

Data $=c(0.036,0.058,0.061,0.074,0.078,0.086,0.102$, $0.103,0.114$,

$0.116,0.148,0.183,0.192,0.254,0.262,0.379,0.381$, $0.538,0.570$,

$0.574,0.590,0.618,0.645,0.961,1.228,1.600,2.006$, 2.054, 2.804,

$3.058,3.076,3.147,3.625,3.704,3.931,4.073,4.393$, $4.534,4.893$,

$6.274,6.816,7.896,7.904,8.022,9.337,10.940,11.020$, $13.880,14.730,15.080)$

\#\#\#\#\#\#\#\#\#\#\#\#\#\#\#\#\#\#\#\#\#\#\#\#\#\#\#\#\#\#\#\#\#

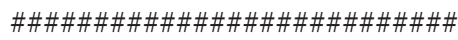

\#\#\#\#\#\#\#\#\#\#\#\#\# pdf of the proposed model

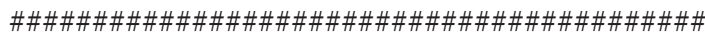
\#\#\#\#\#\#\#\#\#\#\#\#\#\#\#\#\#\#\#\#\#

pdf_pm $<$ - function(par,x) 


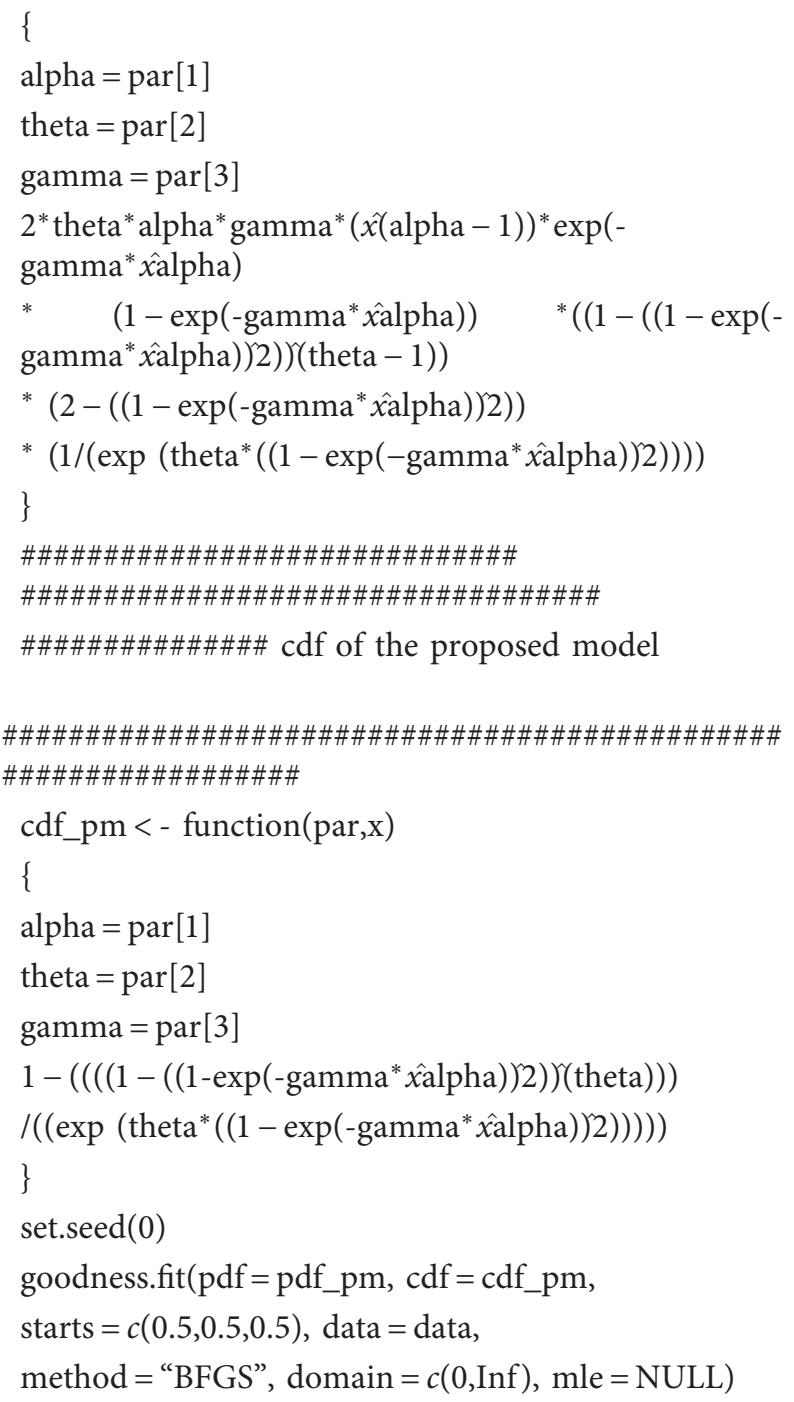

\section{Data Availability}

The data used to support the findings of this study are provided within the article.

\section{Conflicts of Interest}

The authors declare that there are no conflicts of interest regarding the publication of this paper.

\section{Acknowledgments}

This study was supported by Yazd University, Iran.

\section{References}

[1] A. M. Sarhan and M. Zaindin, "Modified weibull distribution. APPS," Applied Sciences, vol. 11, pp. 123-136, 2009.

[2] G. O. Silva, E. M. M. Ortega, and G. M. Cordeiro, "The beta modified Weibull distribution," Lifetime Data Analysis, vol. 16, no. 3, pp. 409-430, 2010.

[3] S. J. Almalki and J. Yuan, "A new modified Weibull distribution," Reliability Engineering \& System Safety, vol. 111, pp. 164-170, 2013.
[4] G. M. Cordeiro, E. M. M. Ortega, and G. Silva, "The beta extended Weibull family," Journal of Probability and Statistical Science, vol. 10, no. 10, pp. 15-40, 2012.

[5] M. Bourguignon, R. B. Silva, and G. M. Cordeiro, "The Weibull-G family of probability distributions," Journal of Data Science, vol. 12, no. 1, pp. 53-68, 2014.

[6] Z. Ahmad, M. Elgarhy, and G. G. Hamedani, "A new Weibull$\mathrm{X}$ family of distributions: properties, characterizations and applications," Journal of Statistical Distributions and Applications, vol. 5, no. 1, pp. 1-18, 2018.

[7] Z. Ahmad, E. Mahmoudi, G. G. Hamedani, and O. Kharazmi, "New methods to define heavy-tailed distributions with applications to insurance data," Journal of Taibah University for Science, vol. 14, no. 1, pp. 359-382, 2020.

[8] A. Alzaatreh, C. Lee, and F. Famoye, "A new method for generating families of continuous distributions," Metron, vol. 71, no. 1, pp. 63-79, 2013.

[9] M.-H. Chen and Q.-M. Shao, "Monte Carlo estimation of Bayesian credible and HPD intervals," Journal of Computational and Graphical Statistics, vol. 8, no. 1, pp. 69-92, 1999.

[10] S. Nasiru, P. N. Mwita, and O. Ngesa, "Exponentiated generalized Transformed Transformer family of distributions," Journal of Statistical and Econometric Methods, vol. 6, no. 4, pp. 1-17, 2017.

[11] I. S. Gradshteyn and I. M. Ryzhik, Tables of Integrals, Series and Products, Academic Press, New York, NY, USA, 2007.

[12] M. Alizadeh, M. Rasekhi, H. M. Yousof, and G. G. Hamedani, "The transmuted Weibull G family of distributions," Hacettepe Journal of Mathematics and Statistics, Forthcoming. vol. 47, no. 6, pp. 1671-1689, 2018.

[13] G. Aryal and I. Elbatal, "On the exponentiated generalized modified Weibull distribution," Communications for Statistical Applications and Methods, vol. 22, no. 4, pp. 333-348, 2015.

[14] G. S. Mudholkar and D. K. Srivastava, "Exponentiated Weibull family for analyzing bathtub failure-rate data," IEEE Transactions on Reliability, vol. 42, no. 2, pp. 299-302, 1993.

[15] A. W. Marshall and I. Olkin, "A new method for adding a parameter to a family of distributions with application to the exponential and Weibull families," Biometrika, vol. 84, no. 3, pp. 641-652, 1997.

[16] W. Stute, W. G. Manteiga, and M. P. Quindimil, "Bootstrap based goodness-of-fit-tests," Metrika, vol. 40, no. 1, pp. 243-256, 1993.

[17] O. Kharazmi, A. Saadatinik, and S. Jahangard, "Odd hyperbolic cosine exponential-exponential (OHC-EE) distribution," Annals of Data Science, vol. 6, no. 4, pp. 765-785, 2019.

[18] S. Geman and D. Geman, "Stochastic relaxation, Gibbs distributions, and the Bayesian restoration of images," IEEE Transactions on Pattern Analysis and Machine Intelligence, vol. 6, pp. 721-741, 1984.

[19] N. Metropolis, A. W. Rosenbluth, M. N. Rosenbluth, A. H. Teller, and E. Teller, "Equation of state calculations by fast computing machines," The Journal of Chemical Physics, vol. 21, no. 6, pp. 1087-1092, 1953.

[20] W. K. Hastings, "Monte Carlo sampling methods using Markov chains and their applications," Biometrika, vol. 57, no. 1, pp. 97-109, 1970.

[21] P. Congdon, Bayesian Statistical Modelling, John Wiley \& Sons, New York, NY, USA, 2001.

[22] C.-E. Lee, S. U. Kim, and S. Lee, "Time-dependent reliability analysis using Bayesian MCMC on the reduction of reservoir storage by sedimentation," Stochastic Environmental Research and Risk Assessment, vol. 28, no. 3, pp. 639-654, 2014. 